August 14, $2021 \quad$ 22:22 WSPC/INSTRUCTION FILE rd2018bV2

\title{
DIRECT SIGNALS FROM ELECTROWEAK SINGLETS THROUGH THE HIGGS PORTAL
}

\author{
RAINER DICK \\ Department of Physics and Engineering Physics, University of Saskatchewan, \\ 116 Science Place, Saskatoon, SK S7N 5E2, Canada \\ rainer.dick@usask.ca
}

\begin{abstract}
We review predictions and constraints for nuclear recoil signals from Higgs portal dark matter under the assumption of standard thermal creation from freeze-out. Thermally created scalar and vector Higgs portal dark matter masses are constrained to be in the resonance region near half the Higgs mass, $m \lesssim m_{h} / 2$, or above several $\mathrm{TeV}$. The resonance region for these models will be tested by XENONnT and LZ. The full mass range up to the unitarity limit can be tested by DarkSide-20k and DARWIN. Fermionic Higgs portal dark matter with a pure CP odd coupling is constrained by the Higgs decay width, but has strongly suppressed recoil cross sections which cannot be tested with upcoming experiments. Fermionic Higgs portal dark matter with a combination of CP even and odd Higgs couplings can be constrained by the direct search experiments.
\end{abstract}

Keywords: Dark matter; Higgs portal; nuclear recoil; direct search.

PACS numbers: 95.35.+d; 12.60.Fr; 14.80.Bn

\section{Introduction}

The existence of a dark sector is firmly established through many different astronomical techniques. It is now widely accepted that cold dark matter forms and sustains large scale structure in the universe because the amount of baryonic matter is severely constrained by the successful theory of big bang nucleosynthesis and by microlensing searches. The existence of dark matter is also directly inferred from galactic rotation curves, motion of galaxies in galaxy clusters, gravitational lensing, gas temperature in galaxy clusters, and from the observational separation of subdominant baryonic mass components and dominant dark halos in cluster collisions $1 \frac{12}{2}$ For indirect evidence for dark matter, we know that baryons alone could not have generated the observed large scale structure in the universe without the dominant gravitational pull and the head start from cold dark matter, which could start to clump together under the influence of gravity well before the primordial plasma recombined to form neutral atoms. The co-existence of the uniformly distributed dark energy component and the cold dark matter halos around galaxies and galaxy clusters is also confirmed through type 1a supernova fits to distance-redshift relations $\$ \sqrt[3]{4}$ and through fits of cosmological parameters to temperature fluctuations in the cosmic microwave background, ${ }^{[5}$ see also Ref. 6 for a recent overview. 
Furthermore, if we make the reasonable assumption that the cold dark matter abundance was generated through the same thermal production mechanisms in the early universe which determined baryonic abundances (after the emergence of matter-anti-matter asymmetry in the baryonic sector) from one common heat bath, we can infer that dark matter and baryonic matter should not only interact gravitationally, but also e.g. through weak particle interactions. It then appears likely that we should also directly detect dark matter particles in particle physics laboratories, either through production from standard matter collisions at the Large Hadron Collider or future accelerators, or through nuclear recoil searches at underground labs like SNOLab, the Laboratori Nazionali del Gran Sasso, the Sanford Underground Research Facility, or the China JinPing Underground Laboratory.

Dark matter research therefore holds the promise to provide us with a window into particle physics beyond the Standard Model. From a theoretical perspective this begs the question: What do we expect from particle physics beyond the Standard Model? We can try to connect dark matter theory with theoretical attempts to solve other contemporary problems of fundamental physics, e.g. the problem of quantum gravity or the problem to explain the huge difference between the electroweak scale and the Planck scale. We can therefore try to identify candidates for dark matter in string theory, e.g. in the gravitational sector or the hidden $E_{8}$ sector of heterotic string theory, or we can connect it to enhanced symmetries in particle physics through supersymmetric models or Grand Unified Theories or left-right symmetric models. A different approach tries to focus on the problem at hand and only expand the Standard Model with a minimal number of additional helicity states to explain dark matter. These models are known as minimal dark matter models. However, extending the Standard Model with a small number of helicity states to account for dark matter generically will not imply stability of this extended Standard Model up to the Planck scale, although Higgs portal couplings can improve the stability properties of the Standard Model ${ }^{7[15}$ Nevertheless, an appealing property of minimal models (besides Occam's razor) is their predictive power and ensuing testability in particle physics experiments. Indeed, we will see that results from ATLAS, CMS, XENON1T, LUX, and PandaX-II put already very strong constraints on several minimal Higgs portal models if we assume standard thermal freeze-out. Besides being testable in direct and collider based search experiments, the predictive power of these models is also appealing from the discovery perspective: The direct relation between dark matter mass and Higgs portal coupling also implies that any potential signal from direct search experiments determines both the coupling and the mass of the dark matter particles. The signal can therefore not only be tested by other direct search experiments (and in the low mass sector also by ATLAS and CMS), but also by gamma-ray and neutrino telescopes by focusing on the energy range of annihilation products corresponding to the proposed dark matter mass.

It was in the framework of the minimal models that attention turned in particular to virtual Higgs exchange as a promising coupling between dark matter and baryons and where the phrase Higgs portal for such a coupling was coined ${ }^{\sqrt{16}}$ How- 
ever, Higgs exchange is not limited to minimal dark matter models, but can also occur in many of the more elaborate models.

Indeed, it is very natural to expect virtual Higgs exchange to contribute to interactions between baryons and dark matter. With mounting evidence that neutrinos also have mass, it appears increasingly likely that the Higgs particle couples already at tree-level to every Standard Model particle except photons and gluons. Therefore, besides gravity, Higgs exchange is the only known interaction which affects almost every known particle directly through Yukawa couplings. While neither necessary nor unavoidable, the assumption that dark matter particles also couple to the Higgs field certainly appears natural, and it is these kinds of models and their prospects for direct search experiments, which are the focus of this review.

Over the years numerous thermal and non-thermal mechanisms have been proposed for the generation of dark matter in the early universe. Creation through a Higgs portal can e.g. play a role in the freeze-in of dark matter components which are interacting so weakly that they are never thermalized ${ }^{[7]}$ Furthermore, Higgs portal interactions can contribute to the interactions of asymmetric dark matter ${ }^{30 \mid} 42$ Thermal freeze-out from a heat bath is a generic mechanism for creating relic matter abundances, $\stackrel{43,45}{4}$ and the question arises whether it is the dominant or a sub-dominant mechanism for the creation of dark matter in the early universe. Thermal freeze-out would e.g. be negligible if the coupling of the potential dark matter component is too large, which would result in too large of a thermally averaged annihilation cross section 46

$$
\langle\sigma v\rangle(g, T)=\frac{1}{8 m^{4} T K_{2}^{2}(m / T)} \int_{4 m^{2}}^{\infty} d s \sqrt{s}\left(s-4 m^{2}\right) \sigma(s) K_{1}(\sqrt{s} / T)
$$

of the component. Here $s$-channel domination is assumed and the dependence on dark matter couplings $g$ is implicit in $\sigma(s)$. Large annihilation cross section means late decoupling and low remnant freeze-out density $\varrho \propto\langle\sigma v\rangle^{-1}$ of the component. In that case the component has to be generated by other means, e.g. through phase transitions or coherent oscillations, to become a viable dark matter candidate.

Mass constraints from direct detection limits can be relaxed in models with effective Sommerfeld enhancement of the annihilation cross sections, $\frac{47,49}{46}$ or through co-annihilation terms which prevent early freeze-out for small dark matter coupling $\sqrt[50]{52]}$ However, this is not the case in the minimal $\mathbb{Z}_{2}$ symmetric Higgs portal models. Here we will define a minimal Higgs portal model as a model where standard thermal freeze-out is the relevant mechanism for dark matter creation, and all assertions about experimentally ruled out or permitted dark matter mass values are only valid under that premise.

Thermal freeze-out models for dark matter are constrained by unitarity $\sqrt{53}$ to dark matter masses $m_{D} \lesssim 100 \mathrm{TeV}$. This arises from the fact that unitarity of the scattering matrix in partial wave expansion implies a bound on the total scattering 
cross section,

$$
\sigma \leq \frac{4 \pi}{k^{2}\left(2 s_{1}+1\right)\left(2 s_{2}+1\right)} \sum_{\ell=0}^{\infty} \sum_{s=\left|s_{1}-s_{2}\right|}^{s_{1}+s_{2}} \sum_{j=|\ell-s|}^{\ell+s}(2 j+1),
$$

where $s_{1}$ and $s_{2}$ are the spins of the two incoming particles. In particular, the bound on $s$-wave dominated annihilation cross sections is $\sigma^{(\ell=0)} \leq 4 \pi / k^{2}$, and this yields in the non-relativistic limit

$$
v \sigma^{(\ell=0)} \leq \frac{4 \pi}{m_{D}^{2} v} .
$$

This upper limit implies for the low-energy dominated thermally averaged annihilation cross section of the dark matter particles the estimate $\langle v \sigma\rangle \lesssim\left(4 \pi / m_{D}^{2}\right)\left\langle v^{-1}\right\rangle$. On the other hand, the required thermally averaged annihilation cross section for generating the observed cold dark matter abundance from freeze-out $\langle v \sigma\rangle \lesssim$ $3 \times 10^{-26} \mathrm{~cm}^{3} / \mathrm{s}$ varies very weakly with mass. Estimates for the parameter $\left\langle v^{-1}\right\rangle$ then yield the upper limit ${ }^{53} m_{D} \lesssim 100 \mathrm{TeV}$.

Furthermore, the requirement of perturbativity can imply additional constraints on the high mass values, since the required thermal cross section $\langle\sigma v\rangle_{f}$ for dark matter freeze-out varies only logarithmically with mass, whereas the actual thermally averaged annihilation cross section $\langle\sigma v\rangle\left(g, T_{f}\right)$ is suppressed for large dark matter masses. This implies that the required dark matter couplings $g^{2}=\langle\sigma v\rangle_{f} /\langle\sigma v\rangle\left(1, T_{f}\right)$ increase with dark matter mass, and for the bosonic models they eventually reach the (conventionally defined) non-perturbative limit $g \simeq 4 \pi$ before the unitarity limit is reached. This happens at masses of several ten $\mathrm{TeV}$, and means that perturbative calculations of recoil cross sections cannot be trusted above the perturbativity mass limits and should only be considered as order of magnitude estimates near the unitarity limit for the bosonic models. The estimates are nevertheless interesting guide posts for the power of current and future direct search experiments to push particle physics to the unitarity limit, and therefore we will also display those leading order estimates for the bosonic recoil cross sections.

The sensitivities of the Argon and Xenon based direct search experiments are extrapolated into the high mass regime, usually up to $10 \mathrm{TeV}$ for XENON1T, and up to the unitarity limit $m_{D} \lesssim 100 \mathrm{TeV}$ for future experiments. Far above the detector threshold, the number of recoil events scales with dark matter mass like $m_{D}^{-1} S\left(q\left(m_{D}\right)\right)$ such that exclusion limits on the basis of the same number of expected events scale like $m_{D} S^{-1}\left(q\left(m_{D}\right)\right)$. The first factor is due to the reduced dark particle flux $j=\varrho_{D} v_{D} / m_{D}$. The second factor is due to the decrease of the nuclear structure factor ${ }^{55} 56 \quad S(q)=F^{2}(q)$ with momentum, which is taken into account through a Helm structure factor. The maximal nuclear recoil energy and maximal momentum transfer in Xenon are $E_{r} \lesssim 175 \mathrm{keV}$ and $Q \lesssim 200 \mathrm{MeV} / c$, respectively. The minimal exchanged Higgs wavelength is $\lambda \gtrsim 6 \mathrm{fm}$, such that the virtual Higgs quanta still probe the nuclei. Actual mass reconstruction for very heavy dark matter from a recoil signal will be challenging ${ }^{[57}$ because the momentum transfer $q$ depends 
on $m_{D}$ only through the reduced mass of the scattering partners, but this does not prevent direct search experiments from pushing the limits far beyond $10 \mathrm{TeV}$, and indeed all the way up to the Planck scale. $\frac{58}{}$

The theory of the effective Higgs nucleon coupling and the impact of strangeness in the nucleon will be reviewed in Sec. 2. Scalar, vector, and fermionic Higgs portal models will then be reviewed in Secs. 3 and 5 , respectively.

\section{The Higgs-nucleon coupling}

Higgs portals entail that dark matter interacts with ordinary matter through Higgs exchange. Shifman, Vainshtein and Zakharov ${ }^{59}$ had demonstrated that the effective Higgs-nucleon coupling $g_{h N} h \bar{N} N$ has at least a strength

$$
g_{h N} v_{h} \simeq 210 \mathrm{MeV}
$$

where $v_{h}=246 \mathrm{GeV}$ is the vacuum expectation value of the Higgs field. The Higgsnucleon coupling is therefore much larger than the Higgs-electron coupling $g_{h e} v_{h}=$ $511 \mathrm{keV}$. Since recoil cross sections for dark matter mass $m_{D}$, Higgs-to-dark matter coupling $g_{h D}$, and Higgs-to-standard particle $P$ coupling $g_{h P}$ are of order $\sigma_{\text {rec }} \simeq$ $g_{h D}^{2} g_{h P}^{2} v_{h}^{2} m_{P}^{2} / \pi m_{h}^{4}\left(m_{D}+m_{P}\right)^{2}$, only nucleon recoils are relevant for Higgs portal dark matter.

However, the Higgs-nucleon coupling depends critically on the strangeness content of the nucleon, which is often expressed in terms of the $y$-parameter

$$
y_{N}=\frac{2\langle N|\bar{s} s| N\rangle}{\langle N|\bar{u} u+\bar{d} d| N\rangle}=\frac{m_{u}+m_{d}}{m_{s}} \frac{\sigma_{s N}}{\sigma_{\pi N}} \simeq \frac{m_{u}+m_{d}}{m_{s}} \frac{f_{s}^{N}}{f_{u}^{N}+f_{d}^{N}}
$$

where

$$
\sigma_{s N}=m_{s}\langle N|\bar{s} s| N\rangle=m_{N} f_{s}^{N}
$$

and

$$
\sigma_{\pi N}=\frac{m_{u}+m_{d}}{2}\langle N|\bar{u} u+\bar{d} d| N\rangle \simeq m_{N}\left(f_{u}^{N}+f_{d}^{N}\right)
$$

are known as $\sigma$-parameters of the nucleon and $m_{N}$ is the nucleon mass. The parameters $f_{q}^{N}$ are defined as $f_{q}^{N}=m_{q}\langle N|\bar{q} q| N\rangle / m_{N}$.

Shifman, Vainshtein and Zakharov had used the fact that the $\sigma$-parameter $\sigma_{\pi N}$ is much smaller than the nucleon mass $m_{N}$ and worked under the assumption of negligible strangeness in the nucleon, $y_{N} \ll 1$. In this case the effective Higgsnucleon coupling is dominated by the coupling of the Higgs to the three heavy quark species charm, bottom and top,

$$
\left.g_{h N} v_{h}\right|_{y_{N} \ll 1} \simeq \sum_{Q=c, b, t} m_{Q}\langle N|\bar{Q} Q| N\rangle,
$$

where the heavy condensates arise from virtual fluctuations of the gluon sea in the nucleon. However, the strangeness content of the nucleon is still not very well known and can have an appreciable effect on the effective Higgs-nucleon coupling. 
This directly affects the predictions for direct dark matter signals, $\underline{60}[63$ as explained in Eq. (14) below.

Cheng 64 e.g. had argued for a large scalar strange form factor $y_{N} \simeq 0.47$. This would increase the effective Higgs-nucleon coupling to $g_{h N} v_{h}=530 \mathrm{MeV}$ because the relatively strong coupling of the Higgs to the strange quark overcompensates for the reduction of the heavy quark condensates which in turn arises from a reduction of the gluon content. Cheng's estimate would increase nuclear recoil cross sections of Higgs portal dark matter by more than a factor of 6 , and would rule out much larger mass ranges in particular for the bosonic Higgs portal models. However, there is wide consensus now that $y_{N}$ is small. We will follow and update the recent analysis performed in Ref. 62, and find a (very cautious) current uncertainty of order 2 in Higgs portal recoil cross sections.

The coupling of the Higgs field to the quarks in the nucleon yields an effective Higgs-nucleon coupling in the form

$$
g_{h N} v_{h}=\sum_{q=u, d, s} m_{q}\langle N|\bar{q} q| N\rangle+\sum_{Q=c, b, t} m_{Q}\langle N|\bar{Q} Q| N\rangle .
$$

On the other hand, the trace anomaly leads to an equation for the nucleon mass in terms of quark and gluon content, $\frac{59}{5}$

$$
m_{N}=\sum_{q=u, d, s} m_{q}\langle N|\bar{q} q| N\rangle+\sum_{Q=c, b, t} m_{Q}\langle N|\bar{Q} Q| N\rangle-\frac{7 \alpha_{s}}{8 \pi}\left\langle N\left|G^{2}\right| N\right\rangle,
$$

while effective heavy quark theory yields for the heavy quark species

$$
m_{Q}\langle N|\bar{Q} Q| N\rangle=-\frac{\alpha_{s}}{12 \pi}\left\langle N\left|G^{2}\right| N\right\rangle .
$$

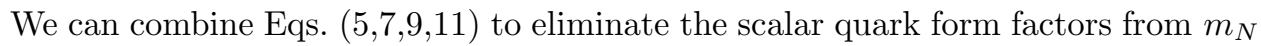
and $g_{h N} v_{h}$,

$$
m_{N} \simeq \sigma_{\pi N}+\frac{m_{s} y_{N}}{m_{u}+m_{d}} \sigma_{\pi N}-\frac{9 \alpha_{s}}{8 \pi}\left\langle N\left|G^{2}\right| N\right\rangle
$$

and

$$
g_{h N} v_{h} \simeq \sigma_{\pi N}+\frac{m_{s} y_{N}}{m_{u}+m_{d}} \sigma_{\pi N}-\frac{\alpha_{s}}{4 \pi}\left\langle N\left|G^{2}\right| N\right\rangle .
$$

This yields an expression for the effective Higgs-nucleon coupling $g_{h N} v_{h}$ in terms of $\sigma_{\pi N}, y_{N}$, and masses, $\underline{62}$

$$
\begin{aligned}
g_{h N} v_{h} & \simeq \frac{7}{9}\left(1+\frac{m_{s} y_{N}}{m_{u}+m_{d}}\right) \sigma_{\pi N}+\frac{2}{9} m_{N} \\
& \simeq \frac{7}{9} \sum_{q=u, d, s} m_{N} f_{q}^{N}+\frac{2}{9} m_{N} \equiv m_{N} f_{N} .
\end{aligned}
$$

The assumptions of Shifman, Vainshtein and Zakharov imply negligible contributions from the light quarks, and this leads to their estimate $g_{h N} v_{h} \simeq 2 m_{N} / 9 \simeq 210$ 
$\mathrm{MeV}$. On the other hand, recent result from lattice calculations,,$\sqrt[65]{70}$ chiral perturbation theory ${ }^{71}$ and sum rules ${ }^{72}$ indicate $0 \leq y_{N} \leq 0.1$ and $\sigma_{\pi N} \leq 55 \mathrm{MeV}$. This yields an estimate for the possible range of the effective Higgs-nucleon coupling,

$$
210 \mathrm{MeV} \lesssim g_{h N} v_{h} \lesssim 310 \mathrm{MeV}
$$

and amounts to a factor of 2.2 of uncertainty in the nuclear recoil cross sections of Higgs portal dark matter particles. Furthermore, Durr et al. find a higher strangeness content ${ }^{73} y_{N}=0.20(8)$ with a lower $\sigma_{\pi N}=38(3)(3) \mathrm{MeV}$, where the errors are statistical and systematic. The large $y_{N}$ results from a large $\sigma_{s N}=105(41)(37) \mathrm{MeV}$, and this translates into a large effective Higgs-nucleon coupling (all errors added in quadrature) $g_{h N} v_{h}=320(43) \mathrm{MeV}$. Hoferichter $\frac{63}{6}$ et $a l$. find a coupling equivalent to $g_{h N} v_{h}=289(17) \mathrm{MeV}$ from averaging $f_{q}^{N}$ values and estimating related errors from four of the recent lattice calculations 68 , 70,73 However, the lattice calculations themselves do not have mutually overlapping error bars, e.g. the results of Ref. 70 yield $g_{h N} v_{h}=263(10) \mathrm{MeV}$. Therefore, we are still more comfortable with the use of a possible range of values for $g_{h N} v_{h}$. We also note that the recent evaluations by ATLAS ${ }^{74}$ and $\mathrm{CMS}^{75}$ used large uncertainties of $0.26 \leq f_{N} \leq 0.66$ and $0.260 \leq f_{N} \leq 0.629$, respectively, and that the careful evaluation by Alarcón et al. from pion-nucleon scattering and pionic atomic spectroscopy ${ }^{76}$ yields $\sigma_{\pi N}=59(7) \mathrm{MeV}$ and $g_{h N} v_{h} \simeq 263(66) \mathrm{MeV}$, in good agreement with the conservative range (15).

We therefore use the SVZ reference point $g_{h N} v_{h}=210 \mathrm{MeV}$ for displaying limits from nucleon recoil cross sections for Higgs portal matter, because these provide the least stringent constraints while including the allowed mass ranges for ${ }^{70}\left[76 g_{h N} v_{h}=\right.$ $263 \mathrm{MeV}$ and for the Hoferichter et al. value $g_{h N} v_{h}=289 \mathrm{MeV}$. This is not borne out of a desire to protect any of the testable Higgs portal models from early elimination, but out of caution. Constraining dark matter models from absence of a signal is different from dark matter mass reconstruction from a signal, and should be based on the least constraining available parameter estimates. However, we also report mass limits for an effective Higgs-nucleon coupling $g_{h N} v_{h}=289 \mathrm{MeV}$.

\section{Scalar Higgs Portal Dark Matter}

The coupling $g_{S} S^{2} H^{+} H$ of a scalar electroweak singlet $S$ to the Higgs field $H$ in unitary gauge

$$
H=\left(\begin{array}{c}
\phi^{+} \\
\phi^{0}
\end{array}\right) \rightarrow \frac{v_{h}+h}{\sqrt{2}}\left(\begin{array}{l}
0 \\
1
\end{array}\right)
$$

yields the minimal renormalizable dark matter addition $\mathcal{L}_{S}$ to the Lagrangian of the Standard Model,

$$
\mathcal{L}_{S}=-\frac{1}{2} \partial S \cdot \partial S-\frac{1}{2} m_{S}^{2} S^{2}-\frac{\lambda_{S}}{4} S^{4}-g_{S} v_{h} S^{2} h-\frac{g_{S}}{2} S^{2} h^{2}
$$


This minimal Standard Model extension has been suggested on numerous occasions as a dark matter mode ${ }^{16} 77 \sqrt{81}$ or as a complementary dark sector component 8283 The very small parameter space provides this model with very high predictive power, and implications for indirect dark matter signals 84 and direct signals $52|62| 88|92| 108]$ has been the subject of numerous investigations. The stability poperties of the model and its extension to a complex scalar have been studied in Refs. 17, 8, 9, 10, 11, 12, 13, 14, 15, and have also been studied as a consequence of radiative conformal symmetry breaking in Refs. 109, 110,

Analysis of the correlation between dark matter mass and couplings from thermal creation requires the corresponding dark matter annihilation cross sections. For completeness we recall the leading order contributions for scalar singlet annihilations into Higgs particles, fermions, and gauge bosons,

$$
\begin{gathered}
\sigma_{S S \rightarrow h h}(s)=\frac{g_{S}^{2} \sqrt{s-4 m_{h}^{2}}}{8 \pi s \sqrt{s-4 m_{S}^{2}}}\left(\frac{s+2 m_{h}^{2}}{s-m_{h}^{2}}\right)^{2}, \\
\sigma_{S S \rightarrow f \bar{f}}(s)=N_{c} g_{S}^{2} \frac{{\sqrt{s-4 m_{f}^{2}}}^{3}}{2 \pi s \sqrt{s-4 m_{S}^{2}}} \frac{m_{f}^{2}}{\left(s-m_{h}^{2}\right)^{2}+m_{h}^{2} \Gamma_{h}^{2}},
\end{gathered}
$$

with $N_{c}=1$ for leptons and $N_{c}=3$ for quarks, and

$$
\sigma_{S S \rightarrow Z Z, W^{+} W^{-}}(s)=\frac{g_{S}^{2} \sqrt{s-4 m_{W, Z}^{2}}}{4 \pi s \sqrt{s-4 m_{S}^{2}}\left(1+\delta_{Z}\right)} \frac{\left(s-2 m_{W, Z}^{2}\right)^{2}+8 m_{W, Z}^{4}}{\left(s-m_{h}^{2}\right)^{2}+m_{h}^{2} \Gamma_{h}^{2}} .
$$

Here $\delta_{Z}=1$ for annihilation into $Z$ bosons and $\delta_{Z}=0$ for annihilation into $W^{+} W^{-}$. The velocity weighted cross sections are $v \sigma=2 \sqrt{1-\left(4 m_{S}^{2} / s\right)} \sigma(s)$, and thermal averaging is performed according to (1).

Higgs portal dark matter from thermal freeze-out is constrained by direct search experiments, and in the low-mass sector $m_{S} \lesssim m_{h} / 2 \simeq 62.5 \mathrm{GeV}$ it is also constrained by the limits from the ATLAS and CMS collaborations ${ }^{75}[111$ on the branching ratio into invisible Higgs decays,, $95[99[112 \mid 114$ see also Ref. 115] for a general recent discussion of LHC dark matter searches. These limits directly constrain the mass $m_{S}$ of Higgs portal dark matter, because the requirement of the correct abundance $\varrho_{D}$ of the dark matter particles relates dark matter coupling and mass, $g_{S}=f\left(m_{S}\right)$. The Higgs decay width

$$
\Gamma_{h \rightarrow S S}=\frac{g_{S}^{2} v_{h}^{2}}{8 \pi m_{h}^{2}} \sqrt{m_{h}^{2}-4 m_{S}^{2}}
$$

then implies the constraint $m_{S} \gtrsim 53.3 \mathrm{GeV}$ on scalar Higgs portal dark matter under the constraint ${ }^{75} \mathcal{B}=\Gamma_{h \rightarrow \text { inv. }} /\left(\Gamma_{h \rightarrow \text { inv. }}+\Gamma_{h \rightarrow \mathrm{SM}}\right) \leq 0.24$ on the branching ratio for invisible Higgs decays, see Fig. 1 .

Note that this constraint becomes stronger if Higgs portal matter is not the dominant dark matter component in the universe, because the required coupling 


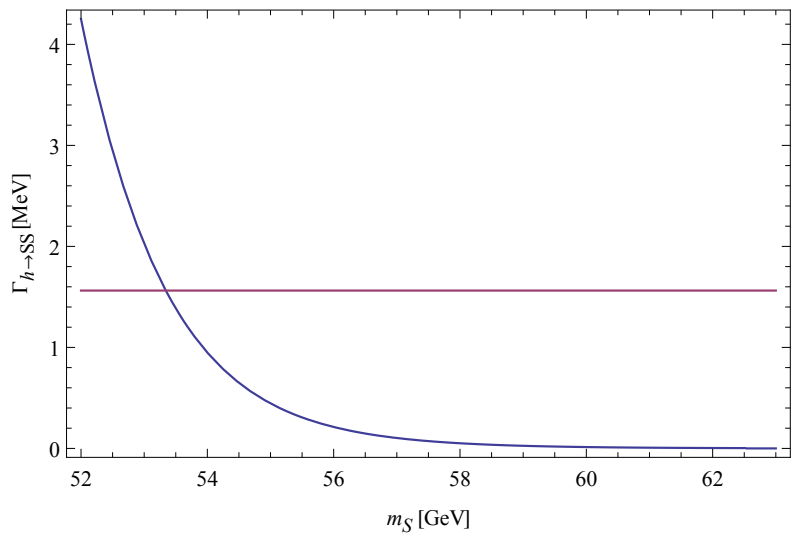

Fig. 1. The invisible decay width $\Gamma_{h \rightarrow S S}$ for $m_{S}$ between $52 \mathrm{GeV}$ and $m_{h} / 2$. The horizontal line arises from the limit on the branching ratio for invisible Higgs decays, $\mathcal{B}=\Gamma_{h \rightarrow \text { inv. }} /\left(\Gamma_{h \rightarrow \text { inv. }}+\right.$ $\left.\Gamma_{h \rightarrow \mathrm{SM}}\right) \leq 0.24$. The Higgs portal coupling $g_{h S}\left(m_{S}\right)$ is determined from the requirement that scalar Higgs portal matter accounts for the observed dark matter.

strength for creating a remnant dark matter density $\varrho_{S}$ from freeze-out of particle annihilation scales like $g_{S}^{2} \propto \varrho_{S}^{-1}$. Larger coupling implies later freeze-out and therefore smaller remnant abundance, but also a larger contribution to the Higgs decay width. Assuming e.g. that remnant scalar Higgs portal matter contributes about $50 \%$ of the dark matter in the universe would increase the mass constraint to $m_{S} \gtrsim 54 \mathrm{GeV}$. On the other hand, if the dark matter particles can annihilate also through alternative channels besides virtual Higgs exchange, then the Higgs portal coupling $g_{S}^{2}$ must only account for a smaller fraction $\zeta_{h S}\langle\sigma v\rangle_{f}, 0 \leq \zeta_{h S}<1$, of the required annihilation cross section $\langle\sigma v\rangle_{f}$ for thermal freeze-out, and this reduces the coupling constraint $g_{S}^{2}=f\left(m_{S}\right)$ to $g_{S}^{2}=\zeta_{h S} f\left(m_{S}\right)$, thus also reducing the mass constraint due to the reduced invisible Higgs decay width $\Gamma_{h \rightarrow S S} \rightarrow \zeta_{h S} \Gamma_{h \rightarrow S S}$. Therefore light dark matter with a Higgs portal is still compatible with invisible Higgs decay constraints if the Higgs portal is not the only connection between the dark matter and the baryonic sector.

The nucleon recoil cross section for perturbatively coupled bosonic Higgs portal matter of mass $m_{D}$ and coupling $g_{D}(D \in\{S, V\}$ for scalar or vector dark matter, respectively) is

$$
\sigma_{D N}=\frac{g_{h N}^{2} g_{D}^{2} v_{h}^{2}}{\pi m_{h}^{4}} \frac{m_{N}^{2}}{\left(m_{D}+m_{N}\right)^{2}} .
$$

Here $m_{N}$ is the nucleon mass and $g_{h N}$ is the coupling constant in the effective Higgs-nucleon coupling term $g_{h N} \bar{N} N h$. While the derivation of this equation is more complicated for the vector model due to the presence of helicity factors, averaging and summation over initial and final state helicities yields the same result as for scalar dark matter.

We use an effective nucleon mass of $m_{N}=930.6 \mathrm{MeV}$ for evaluations of (21) 
and the corresponding formulas for fermionic Higgs portal models discussed in the following sections, because this corresponds to the average nucleon mass in stable or long lived Xenon isotopes. However, our results can also be used for DEAP$3600 \frac{130}{130}$ and DarkSide-20k $\frac{131}{1}$ The average nucleon mass in stable Argon isotopes is $930.4 \mathrm{MeV}$. The relative error of order $2 \times 10^{-4}$ is negligible for current purposes of comparing dark matter models to direct search limits.

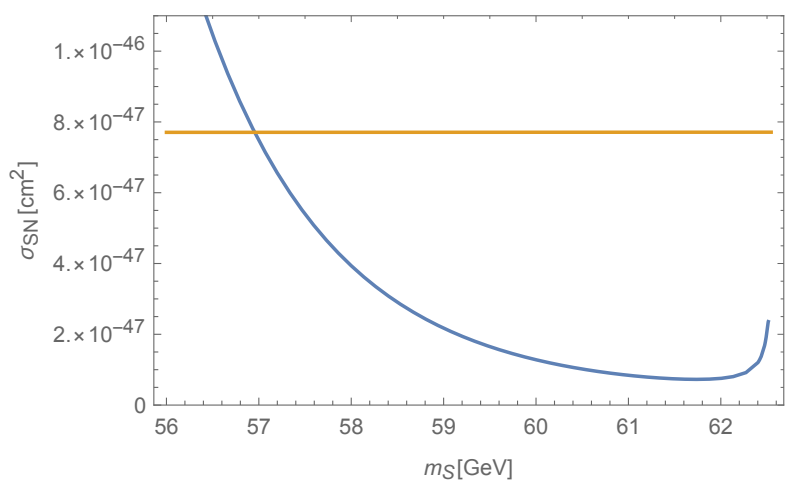

Fig. 2. The nucleon recoil cross section for scalar Higgs portal dark matter in the resonance region $m_{S} \lesssim m_{h} / 2$ for $g_{h N} v_{h}=210 \mathrm{MeV}$. The seemingly horizontal line from XENON1T is actually upwards curved, but varies by less than $1 \%$ in the displayed mass range.

The required small coupling constant $g_{S}$ near the resonance region $m_{S} \lesssim m_{h} / 2$ implies a small recoil cross section in that region which is still compatible with the cross section limits from XENON1T,, 132 which are comparable to but stronger than the limits from PandaX-II ${ }^{133}$ and LUX ${ }^{134}$ In the resonance region, the mass range $57 \mathrm{GeV} \leq m_{S}<m_{h} / 2\left(58 \mathrm{GeV} \leq m_{S}<m_{h} / 2\right.$ for $\left.g_{h N} v_{h}=289 \mathrm{MeV}\right)$ complies with current direct search limits, see Fig. 2 .

The minimal recoil cross section near $m_{S} \simeq 62 \mathrm{GeV}$ is $\sigma_{S N} \simeq 7.3 \times 10^{-48} \mathrm{~cm}^{2}$. Recoil cross sections which are that small can be tested with XENONnT, ${ }^{135}$ LZ, DarkSide-20k ${ }^{131}$ or DARWIN 57

In the high mass sector, extrapolation of the published results from XENON1T rules out scalar Higgs portal dark matter below $m_{S} \lesssim 2.7 \mathrm{TeV}$ for $g_{h N} v_{h}=210$ $\mathrm{MeV}$, see Fig. 3 This is also often displayed in the form of an exclusion region in the $\left(m_{S}, g_{S}\right)$-plane, see the right panel in Fig. 3 , where the yellow region is excluded by the requirement $\Omega_{S} \leq \Omega_{\text {cdm }}$. The excluded mass range above $m_{h} / 2$ increases to $m_{S} \lesssim 4.5 \mathrm{TeV}$ for stronger Higgs-nucleon coupling $g_{h N} v_{h}=289 \mathrm{MeV}$.

In the very high mass region, we can use the perturbative formula 21 as well as the annihilation cross sections (17)-19) only up to a maximal mass value $m_{l D}$ when the dark matter coupling approaches the perturbativity limit $g_{D} \lesssim 4 \pi$. For the scalar model, this limit is reached for $m_{l S} \lesssim 67 \mathrm{TeV}$. Beyond this mass value, the scalar Higgs portal recoil cross section in Fig. 4 should only be considered as an 

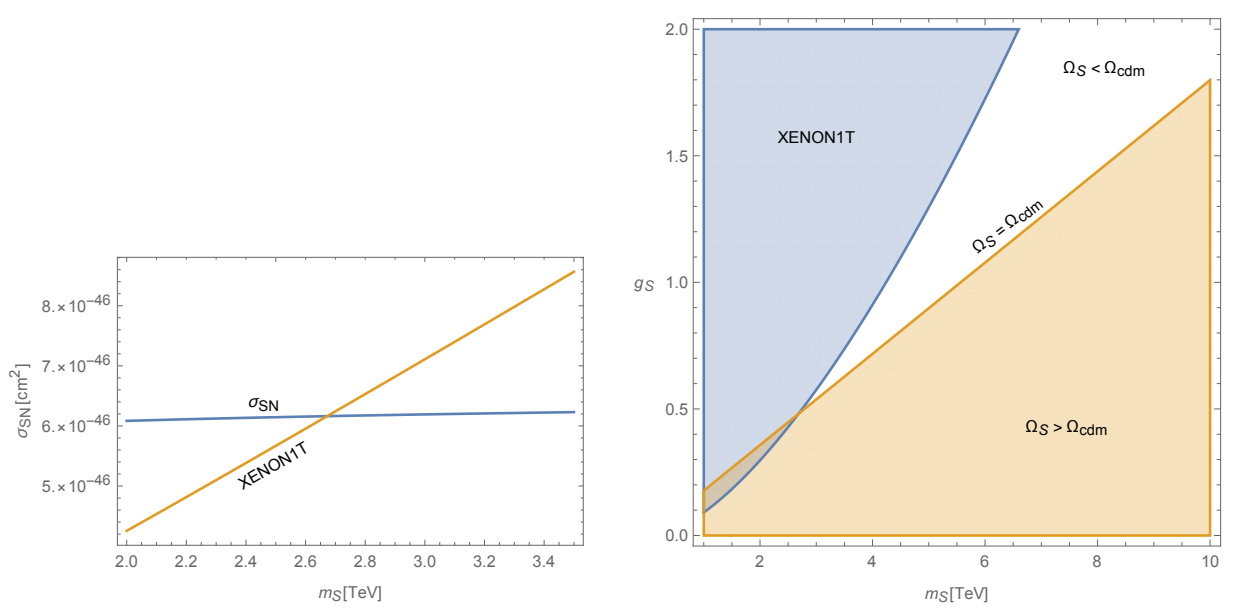

Fig. 3. Left panel: The nucleon recoil cross section for scalar Higgs portal dark matter with $\Omega_{S}=$ $\Omega_{\text {cdm }}$ in the mass range $2 \mathrm{TeV} \leq m_{S} \leq 3.5 \mathrm{TeV}$ versus the extrapolated limit from XENON1T. Right panel: Exclusion regions from XENON1T and the requirement $\Omega_{S} \leq \Omega_{\mathrm{cdm}}$.

order of magnitude estimate. It is nevertheless intriguing that XENONnT and LZ might test scalar Higgs portal dark matter up to about $20 \mathrm{TeV}$, while DarkSide-20k and DARWIN with 200 ton-year exposures could potentially cover the full mass range for frozen-out WIMPS up to the unitarity limit, see Fig. 4 .

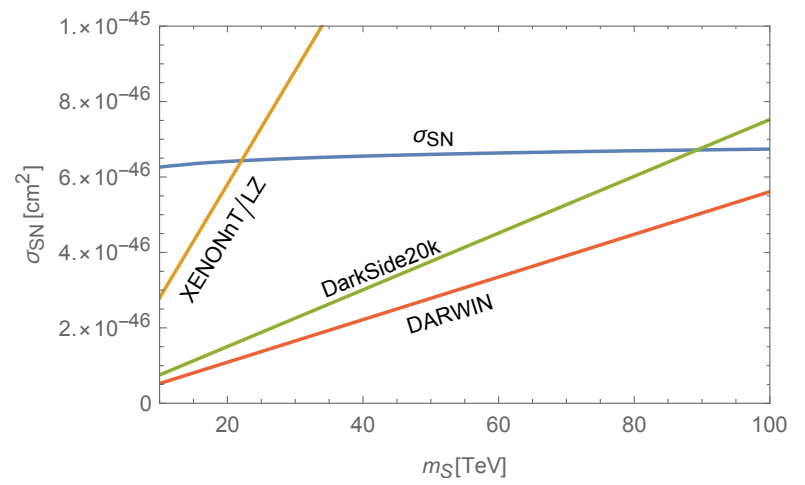

Fig. 4. The estimated nucleon recoil cross section for scalar Higgs portal dark matter in the mass range $10 \mathrm{TeV} \leq m_{S} \leq 100 \mathrm{TeV}$ versus extrapolated sensitivities for XENONnT, LZ, DarkSide-20k and DARWIN. 


\section{Vector Higgs Portal Dark Matter}

Vector Higgs portal dark matter

$$
\begin{gathered}
\mathcal{L}_{V}=-\frac{1}{4} V_{\mu \nu} V^{\mu \nu}-\frac{1}{2} m_{V}^{2} V_{\mu} V^{\mu}-\frac{\lambda_{V}}{4}\left(V_{\mu} V^{\mu}\right)^{2}-g_{V} v_{h} V_{\mu} V^{\mu} h-\frac{g_{V}}{2} V_{\mu} V^{\mu} h^{2} \\
V_{\mu \nu} \equiv \partial_{\mu} V_{\nu}-\partial_{\nu} V_{\mu}
\end{gathered}
$$

can arise in a renormalizable model from spontaneous symmetry breaking in the dark sector $\frac{116}{11}$ and has been further discussed also in Refs. 96, 113, 117, 62, 118, 119, 120, 121, 108, 94. The cross sections for annihilation of the dark vector bosons are

$$
\begin{gathered}
\sigma_{V V \rightarrow h h}=\frac{g_{V}^{2} \sqrt{s-4 m_{h}^{2}}}{288 \pi s \sqrt{s-4 m_{V}^{2}}}\left(\frac{s+2 m_{h}^{2}}{s-m_{h}^{2}}\right)^{2} \frac{\left(s-2 m_{V}^{2}\right)^{2}+8 m_{V}^{4}}{m_{V}^{4}}, \\
\sigma_{V V \rightarrow f \bar{f}}=N_{c} \frac{g_{V}^{2} m_{f}^{2} \sqrt{s-4 m_{f}^{2}}}{72 \pi m_{V}^{4} s \sqrt{s-4 m_{V}^{2}}} \frac{\left(s-2 m_{V}^{2}\right)^{2}+8 m_{V}^{4}}{\left(s-m_{h}^{2}\right)^{2}+m_{h}^{2} \Gamma_{h}^{2}},
\end{gathered}
$$

and

$$
\begin{aligned}
\sigma_{V V \rightarrow Z Z, W^{+} W^{-}}= & \frac{g_{V}^{2} \sqrt{s-4 m_{W, Z}^{2}}}{144 \pi\left(1+\delta_{Z}\right) s \sqrt{s-4 m_{V}^{2}}} \frac{\left(s-2 m_{W, Z}^{2}\right)^{2}+8 m_{W, Z}^{4}}{\left(s-m_{h}^{2}\right)^{2}+m_{h}^{2} \Gamma_{h}^{2}} \\
& \times \frac{\left(s-2 m_{V}^{2}\right)^{2}+8 m_{V}^{4}}{m_{V}^{4}},
\end{aligned}
$$

These low-energy effective annihilation cross sections for $s \lesssim 8 m_{V}^{2}$ do not satisfy the unitarity constraint of bounded $\lim _{s \rightarrow \infty} s \sigma(s)$, which is a familiar indication of the need of UV completion of the vector Higgs portal model through spontaneous symmetry breaking in the dark sector 116$] 117] 119]$ For the same reason, the $m_{V}{ }^{-4}$ term in the denominators of Eqs. (24) 26) (and also in Eq. (30) below) seems to appear unphysical, but is a well-known consequence of averaging over three massive (instead of two massless) polarization states $62|108| 118 \mid 119]$ We can use polarization vectors for the choice $\boldsymbol{e}_{z} \| \boldsymbol{p}$,

$$
\begin{aligned}
& \epsilon^{(1)}(\boldsymbol{p})=(0,1,0,0), \quad \epsilon^{(2)}(\boldsymbol{p})=(0,0,1,0), \\
& \epsilon^{(3)}(\boldsymbol{p})=\frac{1}{m_{V}}\left(|\boldsymbol{p}|, 0,0, \sqrt{\boldsymbol{p}^{2}+m_{V}^{2}}\right) .
\end{aligned}
$$

The sum over the initial polarization states yields

$$
\sum_{\alpha} \epsilon^{(\alpha)}(\boldsymbol{p}) \otimes \epsilon^{(\alpha)}(\boldsymbol{p})=\left(\begin{array}{cccc}
\boldsymbol{p}^{2} / m_{V}^{2} & 0 & 0 & |\boldsymbol{p}| \sqrt{\boldsymbol{p}^{2}+m_{V}^{2}} / m_{V}^{2} \\
0 & 1 & 0 & 0 \\
0 & 0 & 1 & 0 \\
|\boldsymbol{p}| \sqrt{\boldsymbol{p}^{2}+m_{V}^{2}} / m_{V}^{2} & 0 & 0 & \left(\boldsymbol{p}^{2}+m_{V}^{2}\right) / m_{V}^{2}
\end{array}\right)
$$


i.e.

$$
\sum_{\alpha} \epsilon^{(\alpha) \mu}(\boldsymbol{p}) \otimes \epsilon^{(\alpha) \nu}(\boldsymbol{p})=\eta^{\mu \nu}+\frac{p^{\mu} p^{\nu}}{m_{V}^{2}}
$$

This implies

$$
\sum_{\alpha, \beta}\left(\epsilon^{(\alpha)}\left(\boldsymbol{p}_{1}\right) \cdot \epsilon^{(\beta)}\left(\boldsymbol{p}_{2}\right)\right)^{2}=\left(\eta^{\mu \nu}+\frac{p_{1}^{\mu} p_{1}^{\nu}}{m_{V}^{2}}\right)\left(\eta_{\nu \mu}+\frac{p_{2 \nu} p_{2 \mu}}{m_{V}^{2}}\right)=2+\frac{\left(p_{1} \cdot p_{2}\right)^{2}}{m_{V}^{4}}
$$

However, the low energy annihilation cross sections 62 108 $116 \mid 119$ used for the analysis of cosmological implications and constraints of vector Higgs portal models because thermal averaging (1) cuts off the high energy parts with $\exp (-\sqrt{s} / T) \lesssim \exp \left(-25 \sqrt{s} / m_{V}\right)$ near $T=T_{f}$.

The contribution to the Higgs decay width

$$
\Gamma_{h \rightarrow V V}=\frac{g_{V}^{2} v_{h}^{2}}{32 \pi m_{h}^{2}} \sqrt{m_{h}^{2}-4 m_{V}^{2}} \frac{\left(m_{h}^{2}-2 m_{V}^{2}\right)^{2}+8 m_{V}^{4}}{m_{V}^{4}},
$$

implies the constraint $m_{V} \gtrsim 56.3 \mathrm{GeV}$ on vector Higgs portal dark matter using again the limit $\mathcal{B} \leq 0.24$ on the branching ratio into invisible Higgs decays, cf. Fig. 1 for the corresponding constraint for the minimal scalar Higgs portal dark matter. However, just like in the scalar case, the direct search limit is also stronger for vector Higgs portal dark matter. The required small coupling constant $g_{V}$ near the resonance region $m_{V} \lesssim m_{h} / 2$ implies a small recoil cross section in that region which is so far still compatible with the direct search constraints ${ }^{132}$ for $58.4 \mathrm{GeV}<$ $m_{V}<m_{h} / 2$ if $g_{h N} v_{h}=210 \mathrm{MeV}$, see Fig. 5 . A stronger Higgs-nucleon coupling of $g_{h N} v_{h}=289 \mathrm{MeV}$ decreases this mass range to $59.6 \mathrm{GeV}<m_{V}<m_{h} / 2$. The minimal recoil cross section near $m_{V} \simeq 62 \mathrm{GeV}, \sigma_{V N} \simeq 2.1 \times 10^{-47} \mathrm{~cm}^{2}$, will be within reach of XENONnT, LZ, DarkSide-20k and DARWIN.

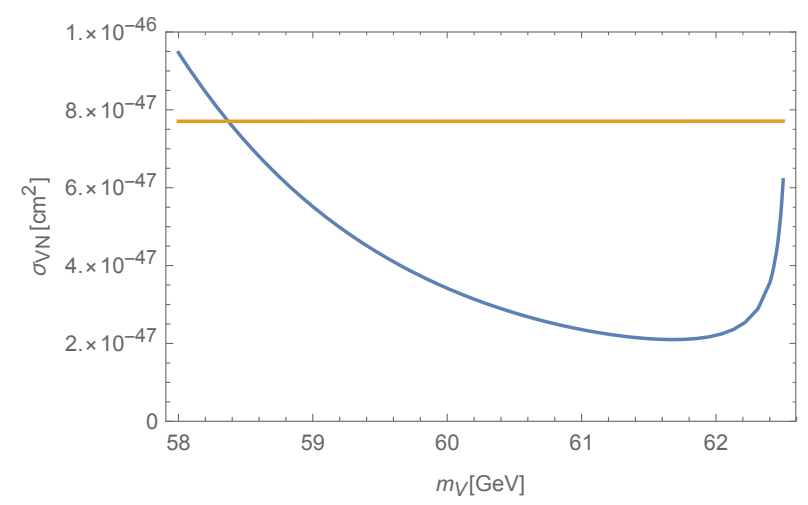

Fig. 5. The nucleon recoil cross section for vector Higgs portal dark matter in the resonance region $m_{V} \lesssim m_{h} / 2$ versus the limits from XENON1T. 

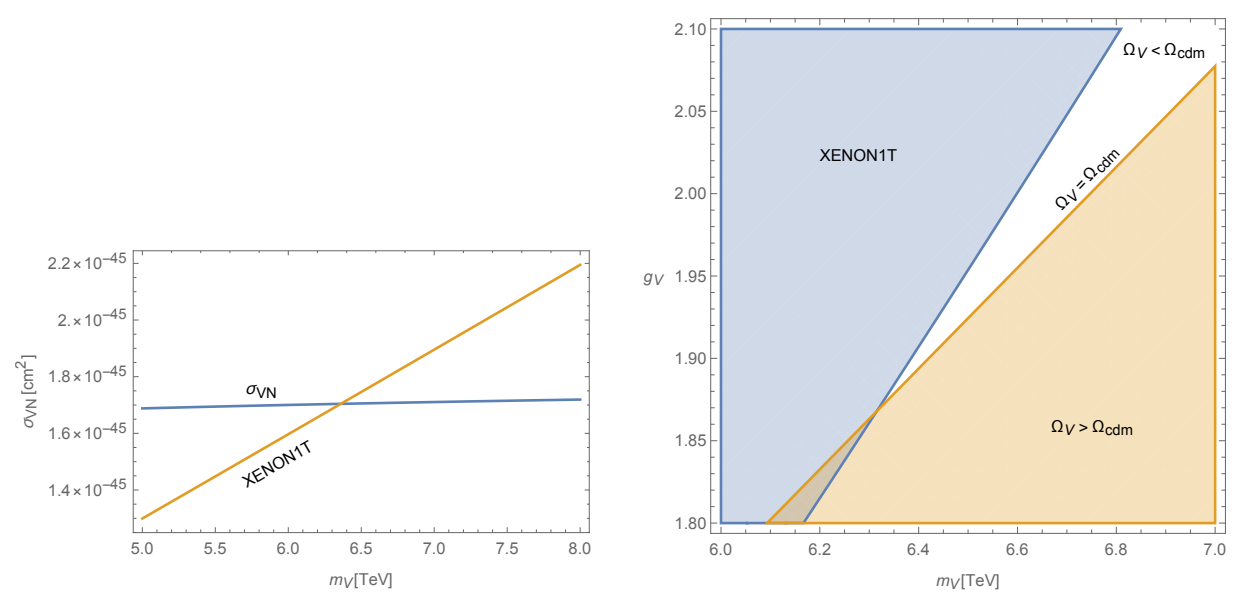

Fig. 6. Left panel: The nucleon recoil cross section for vector Higgs portal dark matter with $\Omega_{V}=$ $\Omega_{\mathrm{cdm}}$ and $g_{h N} v_{h}=210 \mathrm{MeV}$ in the mass range $5 \mathrm{TeV} \leq m_{V} \leq 8 \mathrm{TeV}$ versus the extrapolated limit from XENON1T. Right panel: Exclusion regions in the $\left(m_{V}, g_{V}\right)$ plane from XENON1T and the requirement $\Omega_{V} \leq \Omega_{\mathrm{cdm}}$.

In the high mass sector, extrapolation of the published results from XENON1T rules out vector Higgs portal dark matter below $m_{V} \lesssim 6.4 \mathrm{TeV}$ (or below $m_{V} \lesssim 11.7$ TeV for $g_{h N} v_{h}=289 \mathrm{MeV}$ ), see Fig. 6 .

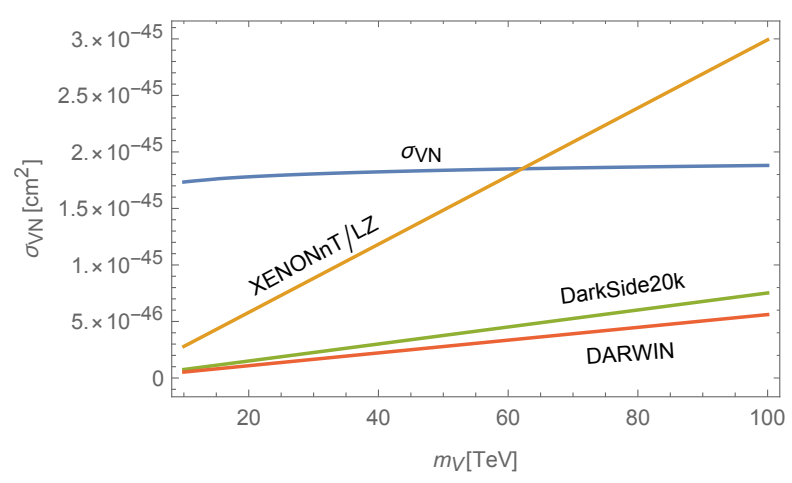

Fig. 7. The nucleon recoil cross section for vector Higgs portal dark matter in the mass range $10 \mathrm{TeV} \leq m_{V} \leq 100 \mathrm{TeV}$ versus the extrapolated sensitivities for XENONnT, LZ, DarkSide-20k and DARWIN.

For yet higher masses, we have to caution that the coupling will reach the perturbativity limit $g_{V} \lesssim 4 \pi$ for a mass limit $m_{l V} \lesssim 40 \mathrm{TeV}$. However, with the caveat that the calculated recoil cross section should at best be considered as an order of magnitude estimate beyond that limit, it is intriguing that XENONnT and LZ may already have the potential to cover the mass range for vector Higgs portal dark 
matter up to about $60 \mathrm{TeV}$ if $g_{h N} v_{h}=210 \mathrm{MeV}$, see Fig. 7. The possible exclusion region would reach the unitarity limit at $100 \mathrm{TeV}$ if $g_{h N} v_{h}=289 \mathrm{MeV}$.

\section{Fermionic Higgs Portal Dark Matter}

Higgs portal couplings of the form

$$
\mathcal{H}=\frac{1}{M} \bar{\chi} \cdot \Gamma \cdot \chi\left(H^{+} \cdot H-\frac{v_{h}^{2}}{2}\right)
$$

to dark fermions $\chi$ can be generated from Yukawa couplings to a scalar mediator $\phi$,

$$
\mathcal{H}_{\phi}=\frac{1}{2} m_{\phi}^{2} \phi^{2}+g \phi \bar{\chi} \cdot \Gamma \cdot \chi+\lambda \phi\left(H^{+} \cdot H-\frac{v_{h}^{2}}{2}\right)
$$

with $M=-m_{\phi}^{2} / g \lambda$. The coupling scale $M$ satisfies $|M|<m_{\phi}$ if $m_{\phi}<|g \lambda|$, i.e. the coupling scale $M$ itself does not necessarily set the scale for new additional degrees of freedom besides the dark matter, and therefore cannot be used to infer a limit on the validity range of the effective Higgs portal couplings (31). These kinds of fermionic Higgs portal models were discussed in Refs. 96, 100, 122, 123, 124, 125, 126, 127, 94, 121, 128, 108. Thermal freeze-out determines the size of the effective Higgs portal coupling $v_{h} / M$ as a function of the dark fermion mass $m_{\chi}$. These models remain perturbative up to the unitarity limit, see e.g. Figs. 9, 10, 12 and 13 below.

\subsection{CP even coupling}

The CP even fermionic Higgs portal model

$$
\begin{aligned}
\mathcal{L}_{\chi} & =\bar{\chi}\left(\mathrm{i} \gamma^{\mu} \partial_{\mu}-m_{\chi}^{(0)}\right) \chi-\frac{1}{M} \bar{\chi} \chi H^{+} \cdot H \\
& =\bar{\chi}\left(\mathrm{i} \gamma^{\mu} \partial_{\mu}-m_{\chi}\right) \chi-\frac{v_{h}}{M} \bar{\chi} \chi h-\frac{1}{2 M} \bar{\chi} \chi h^{2}
\end{aligned}
$$

yields annihilation cross sections

$$
\begin{gathered}
\sigma_{\chi \bar{\chi} \rightarrow h h}(s)=\frac{\sqrt{s-4 m_{\chi}^{2}} \sqrt{s-4 m_{h}^{2}}}{64 \pi M^{2} s}\left(\frac{s+2 m_{h}^{2}}{s-m_{h}^{2}}\right)^{2}, \\
\sigma_{\chi \bar{\chi} \rightarrow f \bar{f}}(s)=N_{c} \frac{\sqrt{s-4 m_{\chi}^{2}}{\sqrt{s-4 m_{f}^{2}}}^{3}}{16 \pi M^{2} s} \frac{m_{f}^{2}}{\left(s-m_{h}^{2}\right)^{2}+m_{h}^{2} \Gamma_{h}^{2}}, \\
\sigma_{\chi \bar{\chi} \rightarrow Z Z, W^{+} W^{-}}(s)=\frac{\sqrt{s-4 m_{\chi}^{2}} \sqrt{s-4 m_{W, Z}^{2}}}{32 \pi M^{2}\left(1+\delta_{Z}\right) s} \frac{\left(s-2 m_{W, Z}^{2}\right)^{2}+8 m_{W, Z}^{4}}{\left(s-m_{h}^{2}\right)^{2}+m_{h}^{2} \Gamma_{h}^{2}} .
\end{gathered}
$$


The corresponding contribution to the Higgs decay width for $m_{\chi}<m_{h} / 2$,

$$
\Gamma_{h \rightarrow \chi \bar{\chi}}=\frac{v_{h}^{2}}{8 \pi M^{2} m_{h}^{2}}{\sqrt{m_{h}^{2}-4 m_{\chi}^{2}}}^{3},
$$

implies the constraint $m_{\chi} \gtrsim 56.2 \mathrm{GeV}$ for $\mathrm{CP}$ even fermionic Higgs portal matter, see Fig. 8 .

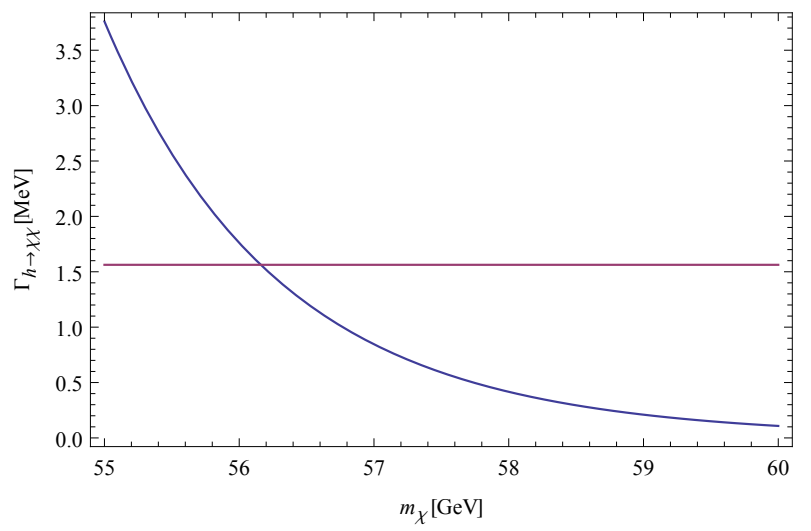

Fig. 8. The invisible decay width $\Gamma_{h \rightarrow \chi \bar{\chi}}$ for the CP even coupling $\sqrt{32}$ and $m_{\chi}$ between $55 \mathrm{GeV}$ and $60 \mathrm{GeV}$. The horizontal line arises from the limit on the branching ratio for invisible Higgs decays, $\mathcal{B}=\Gamma_{h \rightarrow \text { inv. }} /\left(\Gamma_{h \rightarrow \text { inv. }}+\Gamma_{h \rightarrow \mathrm{SM}}\right) \leq 0.24$. The mass parameter $M$ for the $h \chi^{2}$ coupling is determined from the requirement that the fermionic Higgs portal matter accounts for the observed dark matter.

However, whereas for the bosonic models the current direct search constraints in the resonance region were comparable to the Higgs decay constraints, the recoil cross sections of order $\sigma_{\chi N} \simeq 3 \times 10^{-46} \mathrm{~cm}^{2}$ for the model $(32)$ in the region $m_{\chi} \sim m_{h} / 2$ are already ruled out by the direct search experiments.

The coupling scale $M$ can be determined from the requirement that thermal freeze-out of the fermionic Higgs portal matter creates the observed dark matter abundance. It varies in the $1-8 \mathrm{TeV}$ range for dark matter masses in the range $56 \mathrm{GeV} \leq m_{\chi} \leq 63 \mathrm{GeV}$, see Fig. 9 .

The required scale $M$ increases near mass thresholds for $\chi \bar{\chi}$ annihilation, because opening up new annihilation channels implies that thermal freeze-out can produce the observed dark matter abundance with smaller coupling $g_{h \chi}=v_{h} / M$, see Fig. 10 .

Above the top mass, $M$ decreases logarithmically with increasing dark matter mass $m_{\chi}$ because the required cross section $\langle\sigma v\rangle(T)$ for thermal creation decreases with increasing $m_{\chi}$. The decrease in $M$ compensates for the decrease in $\langle\sigma v\rangle(T)$ to ensure that the requirement $\langle\sigma v\rangle\left(T_{f}\right)=\left.\langle\sigma v\rangle_{f} \equiv\langle\sigma v\rangle\right|_{\Omega_{\chi}=\Omega_{\mathrm{cdm}}}$ can still be met. The asymptotic value of $M$ near the unitarity limit $m_{\chi} \simeq 100 \mathrm{TeV}$ is $M \simeq 860 \mathrm{GeV}$.

The property $\sigma_{\chi \bar{\chi}} \propto\left(s-4 m_{\chi}^{2}\right)^{1 / 2}$ of the annihilation cross sections in the CP even fermionic Higgs portal model differs from the corresponding property $\sigma_{D D} \propto$ 


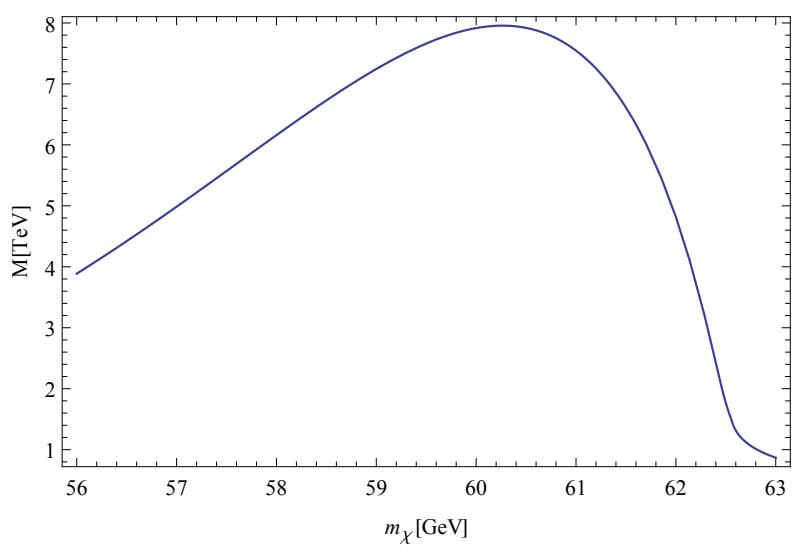

Fig. 9. The required coupling scale $M$ for dark matter creation through the fermionic Higgs portal 32 in the WIMP mass range $56 \mathrm{GeV} \leq m_{\chi} \leq 63 \mathrm{GeV}$.

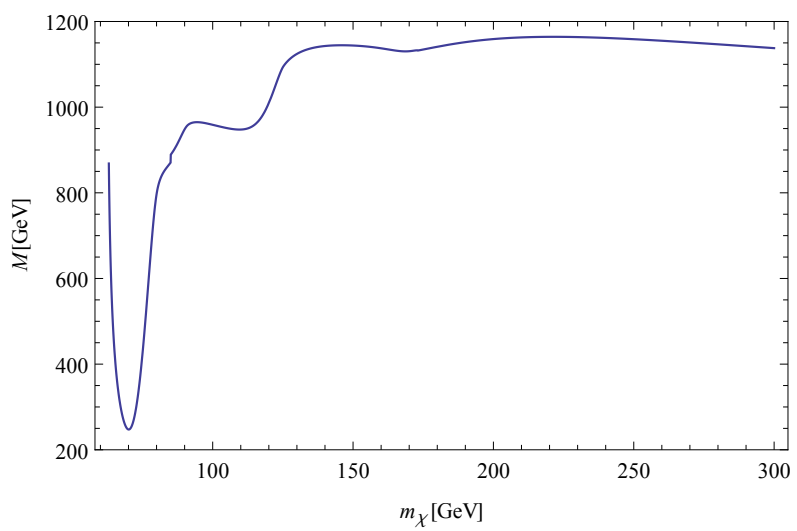

Fig. 10. The required coupling scale $M$ for dark matter creation through the fermionic Higgs portal 32 in the WIMP mass range $63 \mathrm{GeV} \leq m_{\chi} \leq 300 \mathrm{GeV}$.

$\left(s-4 m_{D}^{2}\right)^{-1 / 2}, D \in\{S, V\}$, of the bosonic models. This arises as a consequence of averaging over initial spins in the CP even model $(32)$ (with $k \equiv|\boldsymbol{k}|$ ),

$$
\frac{1}{4} \sum_{s_{1}, s_{2}}\left|\bar{v}\left(-\boldsymbol{k}, s_{2}\right) \cdot u\left(\boldsymbol{k}, s_{1}\right)\right|^{2}=2 k^{2}=\frac{1}{2}\left(s-4 m_{\chi}^{2}\right) .
$$

This leads to thermally averaged cross sections $\langle\sigma v\rangle(T)$ for given coupling $v_{h} / M$ in the $\mathrm{CP}$ even model 32 which are considerably smaller than the corresponding cross sections for given couplings $g_{D}$ in the bosonic models. Comparison with the required cross section $\langle\sigma v\rangle_{f}$ for thermal freeze-out therefore leads to a considerably larger value for $v_{h} / M \gg g_{D}$ for given dark matter mass $m_{\chi}=m_{D}$, and this leads 
to considerably larger recoil cross sections,

$$
\sigma_{\chi N}=\frac{g_{h N}^{2} v_{h}^{2}}{\pi M^{2} m_{h}^{4}}\left(\frac{m_{\chi} m_{N}}{m_{\chi}+m_{N}}\right)^{2},
$$

which are ruled out due to the limits from PandaX-II, LUX and XENON1T even for the weakest conceivable Higgs-nucleon coupling $g_{h N} v_{h}=210 \mathrm{MeV}$, see e.g. Fig. 11 .

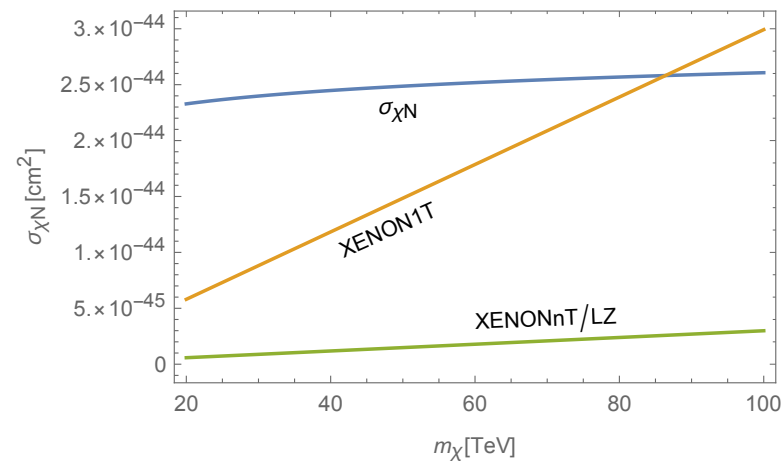

Fig. 11. The recoil cross section for fermionic Higgs portal dark matter with CP even coupling 32 versus the extrapolated XENON1T limit.

However, the CP even model can be combined with the CP odd model in such a way that the recoil cross sections still comply with the direct search limits. This is discussed after the CP odd coupling in Eq. (48) below.

\subsection{CP odd coupling}

Fermionic dark matter with a CP odd Higgs portal,

$$
\begin{aligned}
\mathcal{L} & =\left[\bar{\chi}\left(\mathrm{i} \gamma^{\mu} \partial_{\mu}-m_{\chi}\right) \chi-\frac{\mathrm{i}}{\mu} \bar{\chi} \gamma_{5} \chi\left(H^{+} \cdot H-\frac{v_{h}^{2}}{2}\right)\right]_{h} \\
& =\bar{\chi}\left(\mathrm{i} \gamma^{\mu} \partial_{\mu}-m_{\chi}\right) \chi-\mathrm{i} \frac{v_{h}}{\mu} \bar{\chi} \gamma_{5} \chi h-\frac{\mathrm{i}}{2 \mu} \bar{\chi} \gamma_{5} \chi h^{2},
\end{aligned}
$$

yields annihilation cross sections

$$
\begin{gathered}
\sigma_{\chi \bar{\chi} \rightarrow h h}(s)=\frac{\sqrt{s-4 m_{h}^{2}}}{64 \pi \mu^{2} \sqrt{s-4 m_{\chi}^{2}}} \frac{\left(s+2 m_{h}^{2}\right)^{2}}{\left(s-m_{h}^{2}\right)^{2}}, \\
\sigma_{\chi \bar{\chi} \rightarrow f \bar{f}}(s)=N_{c} \frac{{\sqrt{s-4 m_{f}^{2}}}^{3}}{16 \pi \mu^{2} \sqrt{s-4 m_{\chi}^{2}}} \frac{m_{f}^{2}}{\left(s-m_{h}^{2}\right)^{2}+m_{h}^{2} \Gamma_{h}^{2}},
\end{gathered}
$$




$$
\sigma_{\chi \bar{\chi} \rightarrow Z Z, W^{+} W^{-}}(s)=\frac{\sqrt{s-4 m_{W, Z}^{2}}}{32 \pi \mu^{2}\left(1+\delta_{z}\right) \sqrt{s-4 m_{\chi}^{2}}} \frac{\left(s-2 m_{W, Z}^{2}\right)^{2}+8 m_{W, Z}^{4}}{\left(s-m_{h}^{2}\right)^{2}+m_{h}^{2} \Gamma_{h}^{2}} .
$$

The coupling scale $\mu$ can be determined from the requirement that thermal freeze-out of the fermionic Higgs portal matter creates the observed dark matter abundance. It varies between about $1 \mathrm{TeV}$ and $40 \mathrm{TeV}$ in the mass range $56 \mathrm{GeV} \leq$ $m_{\chi} \leq 63 \mathrm{GeV}$, see Fig. 12

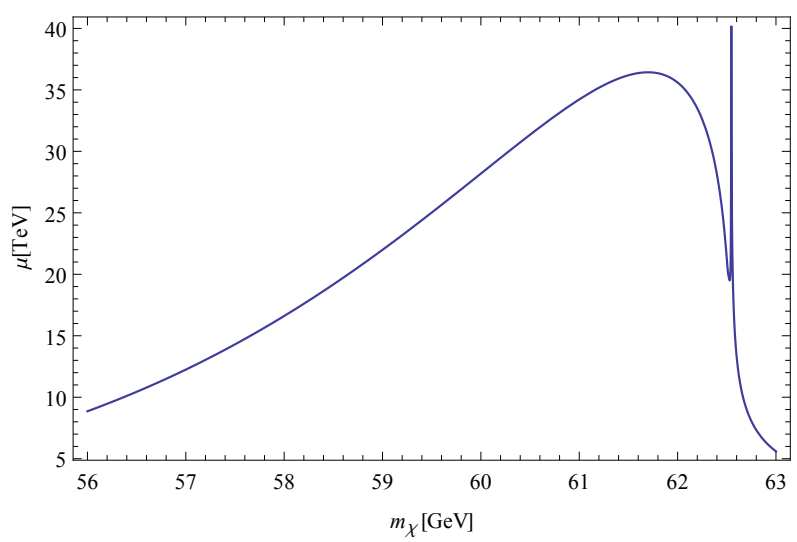

Fig. 12. The required coupling scale $\mu$ for dark matter creation through the fermionic Higgs portal (39) in the WIMP mass range $53 \mathrm{GeV} \leq m_{\chi} \leq 63 \mathrm{GeV}$.

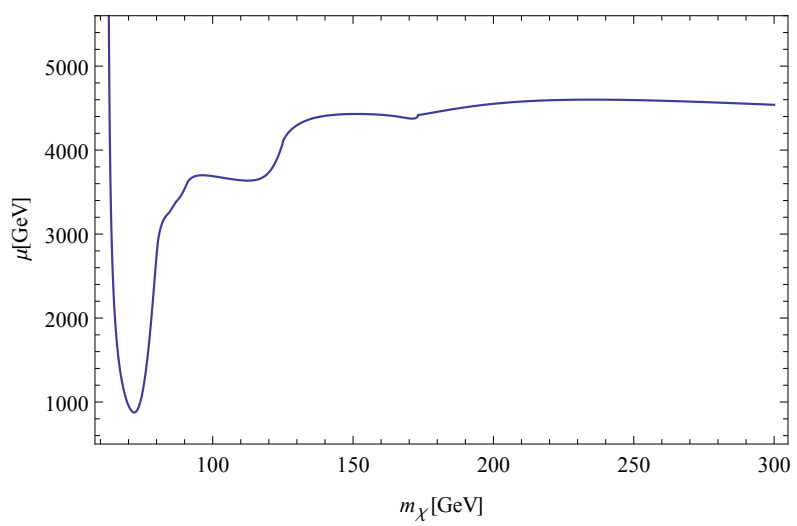

Fig. 13. The required coupling scale $\mu$ for dark matter creation through the fermionic Higgs portal 39 in the WIMP mass range $63 \mathrm{GeV} \leq m_{\chi} \leq 300 \mathrm{GeV}$.

The required scale $\mu$ increases near mass thresholds for $\chi \bar{\chi}$ annihilation, because 
opening up new annihilation channels implies that thermal freeze-out can produce the observed dark matter abundance with smaller coupling $g_{h \chi}=v_{h} / \mu$, see Fig. 13 . Above the top mass, $\mu$ decreases logarithmically with increasing dark matter mass $m_{\chi}$ for the same reason why the scale $M$ increased in the CP even model.

The required coupling scale for the $\mathrm{CP}$ odd model is always larger than the corresponding scale for the $\mathrm{CP}$ even model, $\mu>M$. This is a consequence of the pole near $s=4 m_{\chi}^{2}$ in the annihilation cross sections 40,42 of the CP odd model. The CP odd dark fermion model behaves more like the bosonic models in terms of the pole structure of the annihilation cross sections. The reason for this different behavior of the $\mathrm{CP}$ even and odd fermionic models is that averaging over initial spins yields (37) in the $\mathrm{CP}$ even model $(32)$, whereas for the $\mathrm{CP}$ odd coupling in Eq. (39) we find

$$
\frac{1}{4} \sum_{s_{1}, s_{2}}\left|\bar{v}\left(-\boldsymbol{k}, s_{2}\right) \cdot \gamma_{5} \cdot u\left(\boldsymbol{k}, s_{1}\right)\right|^{2}=2 \omega^{2}(\boldsymbol{k})=s / 2
$$

This also yields the narrow resonance at $m_{\chi}=m_{h} / 2$ in Fig. 12 for the CP odd coupling, which does not appear for the CP even coupling in Fig. 9. The value of $\mu$ near the unitarity limit $m_{\chi} \simeq 100 \mathrm{TeV}$ is $\mu \simeq 3.9 \mathrm{TeV}$.

The nuclear recoil cross section differs from the corresponding result $(38)$ for the parity conserving coupling by a factor $\beta_{\chi}^{2} / 2$, where $0 \leq \beta_{\chi}<1$ is the speed of the dark fermions,

$$
\sigma_{\chi N}=\frac{g_{h N}^{2} v_{h}^{2} m_{N}^{2}}{2 \pi \mu^{2} m_{h}^{4}} \frac{k^{2}}{\left(m_{N}+m_{\chi}\right)^{2}}=\frac{g_{h N}^{2} v_{h}^{2} m_{N}^{2}}{2 \pi \mu^{2} m_{h}^{4}} \frac{\beta_{\chi}^{2} m_{\chi}^{2}}{\left(m_{N}+m_{\chi}\right)^{2}}
$$

Unfortunately, this model is not testable through the direct search experiments in the allowed mass range $56 \mathrm{GeV} \lesssim m_{\chi} \lesssim 100 \mathrm{TeV}$ because $\beta_{\chi} \sim 10^{-3}$ implies that the nuclear recoil cross sections for this model are below the neutrino floor ${ }^{[129}$ for all possible Higgs-nucleon couplings 15 .

This difference between the CP even and odd couplings is again due to the very different behavior under averaging over initial spins and summing over final spins for the recoil events. For the CP even model, the result

$$
\frac{1}{2} \sum_{s, s^{\prime}}\left|\bar{u}\left(\boldsymbol{p}, s^{\prime}\right) \cdot u(\boldsymbol{k}, s)\right|^{2}=\frac{1}{2} \operatorname{tr}[(m-\gamma \cdot p)(m-\gamma \cdot k)]=2\left(m^{2}-p \cdot k\right)
$$

yields a factor $4 m^{2}$ in the non-relativistic limit, whereas for the CP odd coupling the result

$$
\begin{aligned}
\frac{1}{2} \sum_{s, s^{\prime}}\left|\bar{u}\left(\boldsymbol{p}, s^{\prime}\right) \cdot \gamma_{5} \cdot u(\boldsymbol{k}, s)\right|^{2} & =-\frac{1}{2} \operatorname{tr}\left[\gamma_{5} \cdot(m-\gamma \cdot p) \cdot \gamma_{5} \cdot(m-\gamma \cdot k)\right] \\
& =-2\left(m^{2}+p \cdot k\right)
\end{aligned}
$$

yields a factor $(\boldsymbol{p}-\boldsymbol{k})^{2}$ in the non-relativistic limit. 
The small recoil cross sections imply that the constraint from the Higgs decay width for $m_{\chi}<m_{h} / 2$,

$$
\Gamma_{h \rightarrow \chi \bar{\chi}}=\frac{v_{h}^{2}}{8 \pi \mu^{2}} \sqrt{m_{h}^{2}-4 m_{\chi}^{2}},
$$

is significant for this dark matter model and implies $m_{\chi} \gtrsim 56.1 \mathrm{GeV}$, see Fig. 14 .

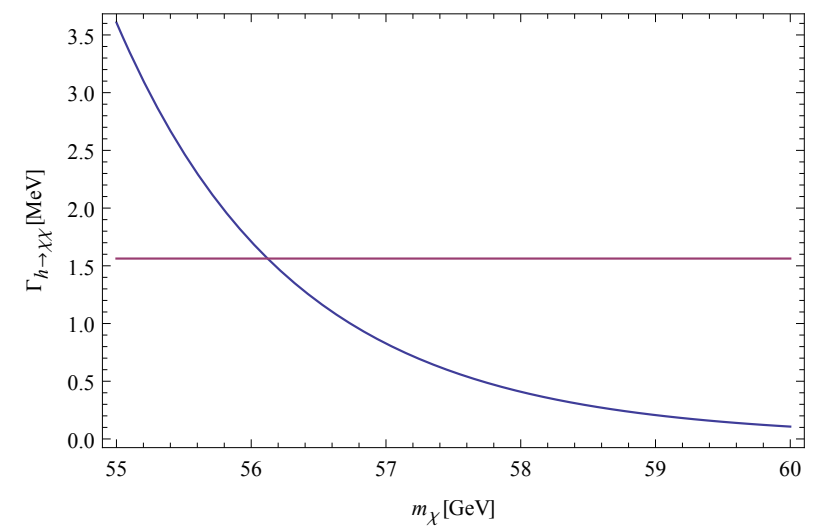

Fig. 14. The invisible decay width $\Gamma_{h \rightarrow \chi \bar{\chi}}$ for the CP odd coupling 39 and $m_{\chi}$ between $55 \mathrm{GeV}$ and $60 \mathrm{GeV}$. The horizontal line arises from the limit on the branching ratio for invisible Higgs decays, $\mathcal{B}=\Gamma_{h \rightarrow \text { inv. }} /\left(\Gamma_{h \rightarrow \text { inv. }}+\Gamma_{h \rightarrow \mathrm{SM}}\right) \leq 0.24$. The mass parameter $M$ for the $h \chi^{2}$ coupling is determined from the requirement that the fermionic Higgs portal matter accounts for the observed dark matter.

The partial decay widths and the resulting mass constraints are virtually identical for the CP even coupling (32) and the CP odd coupling (39) because the relative factor $s /\left(s-4 m_{\chi}^{2}\right)$ between the respective annihilation cross sections $333 \mid 35$ and 440 near $m_{\chi} \lesssim m_{h} / 2$ enters the calculation of the coupling scales $M$ versus $\mu$ and compensates for the relative factor $m_{h}^{2} /\left(m_{h}^{2}-4 m_{\chi}^{2}\right)$ between the partial decay widths (36) and (47). For example, the partial decay width (36) for the CP even model at $m_{\chi}=55 \mathrm{GeV}$ is $\Gamma_{h \rightarrow \chi \bar{\chi}}=3.76 \mathrm{MeV}$, while the corresponding contribution to the Higgs decay width for the CP odd model is $\Gamma_{h \rightarrow \chi \bar{\chi}}=3.61 \mathrm{MeV}$.

The CP even and odd couplings can be combined,

$$
\mathcal{H}_{\chi h}=\bar{\chi} \cdot\left(\frac{1}{M_{\zeta}}+\frac{\mathrm{i}}{\mu_{\zeta}} \gamma_{5}\right) \cdot \chi\left(H^{+} H-\frac{v_{h}^{2}}{2}\right)
$$

with $M_{\zeta}=M / \zeta, \mu_{\zeta}=\mu / \sqrt{1-\zeta^{2}}$, such that the model still complies with the direct search limits.

The $\mathrm{CP}$ even and $\mathrm{CP}$ odd amplitudes do not interfere since spin averaging in the interference terms leaves terms proportional to $\operatorname{tr}\left(\gamma_{5} \gamma_{\mu} \gamma_{\nu}\right)=0, \operatorname{tr}\left(\gamma_{5} \gamma_{\mu}\right)=0$, and $\operatorname{tr}\left(\gamma_{5}\right)=0$. The annihilation cross sections and nulear recoil cross sections are therefore the sums of the cross sections of the $\mathrm{CP}$ even $(+)$ and $\mathrm{CP}$ odd $(-)$ models, 
$\sigma=\zeta^{2} \sigma_{(+)}+\left(1-\zeta^{2}\right) \sigma_{(-)}$, and the same applies to the invisible Higgs decay width, $\Gamma_{h \rightarrow \chi \bar{\chi}}=\zeta^{2} \Gamma_{h \rightarrow \chi \bar{\chi}}^{(+)}+\left(1-\zeta^{2}\right) \Gamma_{h \rightarrow \chi \bar{\chi}}^{(-)}$.

The property for the annihilation cross sections also implies that the required values of the coupling parameters $M\left(m_{\chi}\right)$ and $\mu\left(m_{\chi}\right)$ do not depend on $\zeta$ : The freeze-out requirements for $\zeta=1$, viz. $\langle\sigma v\rangle_{f}=\langle\sigma v\rangle_{(+)}\left(M, T_{f}\right)$, and for $\zeta=0$, viz. $\langle\sigma v\rangle_{f}=\langle\sigma v\rangle_{(-)}\left(\mu, T_{f}\right)$, imply

$$
\langle\sigma v\rangle_{f}=\zeta^{2}\langle\sigma v\rangle_{(+)}\left(M, T_{f}\right)+\left(1-\zeta^{2}\right)\langle\sigma v\rangle_{(-)}\left(\mu, T_{f}\right)
$$

for every value of $\zeta$. $\zeta$ can therefore be directly determined from the requirement that $\sigma_{\chi N}$ complies with the limits from the direct search experiments. Maximal values of $\zeta$ which comply with the XENON1T constraints and for Higgs-nucleon coupling $g_{h N} v_{h}=210 \mathrm{MeV}$ are displayed in Fig. 15. Increasing the Higgs-nucleon coupling to $g_{h N} v_{h}=289 \mathrm{MeV}$ reduces the allowed values of $\zeta$ by a factor 0.727 .

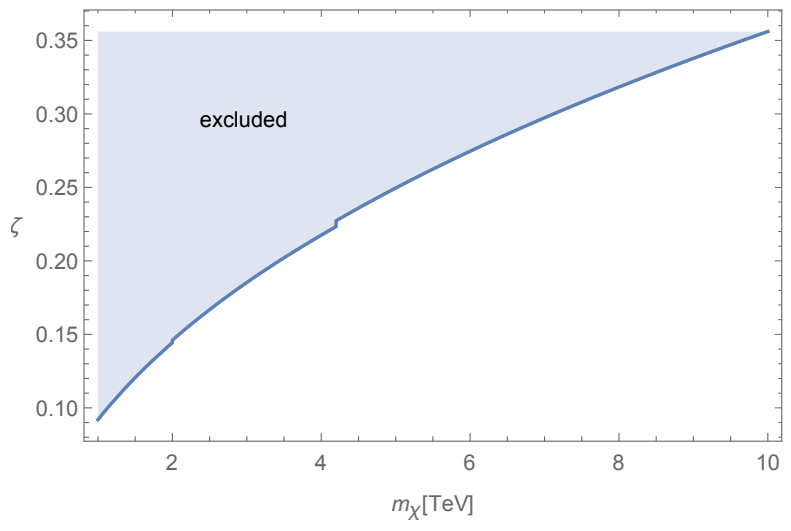

Fig. 15. The maximal values of $\zeta$ for which the Higgs portal coupling 48 still complies with extrapolated XENON1T limits in the mass range between $1 \mathrm{TeV}$ and $10 \mathrm{TeV}$.

For better or worse, this defines a one-parameter Higgs portal model which can always be arbitrary close to the direct search limits, and yet also always be safe from being ruled out by direct search experiments.

\section{Conclusions}

Relic electroweak singlets with a Higgs portal coupling provide an interesting scenario for dark matter due to the direct connection between mass and coupling to baryons, which implies high predictivity and therefore also generically high testability of the models. This in turn also implies easy verification of any potential direct signal through indirect searches.

In particular, the fermionic singlet Higgs portal model with mass suppressed purely $\mathrm{CP}$ even coupling to the Higgs field appears to be ruled out now due to 
the limits from the direct search experiments. Bosonic models are constrained to dark matter masses near the resonance region, $57 \mathrm{GeV} \lesssim m_{D} \lesssim m_{h} / 2$, or to heavy dark matter masses in the few $\mathrm{TeV}$ range or higher. They can be tested up to the unitarity limit by DARWIN and likely also by DarkSide-20k, while XENONnT and LZ will already have the potential to test the bosonic models up to several ten $\mathrm{TeV}$. The fermionic singlet model with pure mass supressed CP odd Higgs coupling is constrained by the limits on the invisible Higgs decay width to masses $m_{\chi} \gtrsim 56$ $\mathrm{GeV}$, but the nuclear recoil cross sections proportional to $\beta_{\chi}^{2}$ are below the neutrino floor and therefore evade the direct search experiments.

The bosonic electroweak singlet Higgs portal models are increasingly constrained by current direct search experiments, and will be further tested by direct search experiments which are currently under construction. Their highly predictive features and their close alignment with current and future sensitivities of direct search experiments make them interesting targets for experimental dark matter research, since any Higgs portal interpretation of a direct detection signal can easily be tested with follow-up cosmic ray observations for the bosonic models. The fermionic Higgs portal with CP odd coupling is an interesting target for collider based searches and indirect searches, but relevance for the direct search experiments requires admixture of a CP even Higgs coupling.

\section{Acknowledgments}

This work was supported by the Natural Sciences and Engineering Research Council of Canada. I would like to thank Jose Alarcón Soriano and Jusak Tandean for very helpful and constructive comments.

\section{References}

1. D. Clowe, A. Gonzalez and M. Markevitch, Astrophys. J. 604 (2004) 596; M. Markevitch, A.H. Gonzalez, D. Clowe, A. Vikhlinin, W. Forman, C. Jones, S. Murray and W. Tucker, Astrophys. J. 606 (2004) 819; D. Clowe, M. Bradač, A.H. Gonzalez, M. Markevitch, S.W. Randall, C. Jones and D. Zaritsky, Astrophys. J. 648 (2006) L109.

2. M. Bradač, S.W. Allen, T. Treu, H. Ebeling, R. Massey, R.G. Morris, A. von der Linden and D. Applegate, Astrophys. J. 687 (2008) 959; W.A. Dawson et al., Astrophys. J. 747 (2012) L42.

3. A.G. Riess et al., Astron. J. 116 (1998) 1009; S. Perlmutter et al., Astrophys. J. 517 (1999) 565.

4. M. Betoule et al., Astron. Astrophys. 568 (2014) A22; A. Rest et al., Astrophys. J. 795 (2014) 44.

5. Planck Collaboration (P.A.R. Ade et al.), Astron. Astrophys. 594 (2016) A13.

6. K. Freese, Int. J. Mod. Phys. D 26 (2017) 1730012.

7. M. Gonderinger, Y. Li, H. Patel and M.J. Ramsey-Musolf, JHEP 1001 (2010) 053.

8. S. Profumo, L. Ubaldi and C. Wainwright, Phys. Rev. D 82 (2010) 123514.

9. M. Gonderinger, H. Lim and M.J. Ramsey-Musolf, Phys. Rev. D 86 (2012) 043511.

10. O. Lebedev, Eur. Phys. J. C 72 (2012) 2058.

11. M. Fabbrichesi and S.T. Petcov, Eur. Phys. J. C 74 (2014) 2774. 
12. V.V. Khoze, C. McCabe and G. Ro, JHEP 1408 (2014) 026.

13. N. Khan and S. Rakshit, Phys. Rev. D 90 (2014) 113008.

14. A. Falkowski, C. Gross and O. Lebedev, JHEP 1505 (2015) 057.

15. I. Garg, S. Goswami, K.N. Vishnudath and N. Khan, Phys. Rev. D 96 (2017) 055020.

16. B. Patt and F. Wilczek, Higgs field portal into hidden sectors, hep-ph/0605188.

17. C.E. Yaguna, JHEP 1108 (2011) 060.

18. M.Frigerio, T. Hambye and E. Masso, Phys. Rev. X 1 (2011) 021026.

19. X. Chu, T. Hambye and M.H.G. Tytgat, JCAP 1205 (2012) 034.

20. A. Merle, V. Niro and D. Schmidt, JCAP 1403 (2014) 028.

21. F.S. Quiroz and K. Sinha, Phys. Lett. B 735 (2014) 69.

22. T. Alanne, K. Tuominen and V. Vaskonen, Nucle. Phys. B 889 (2014) 692.

23. M. Heikinheimo and C. Spethmann, JHEP 1412 (2014) 084.

24. J. McDonald, JCAP 1608 (2016) 035.

25. J. Kim and J. McDonald, A clockwork Higgs portal model for freeze-in dark matter, arXiv:1709.04105 [hep-ph]; Freeze-in dark matter from a sub-Higgs mass clockwork sector via the Higgs portal, arXiv:1804.02661 [hep-ph].

26. M. Duch, B. Grzadkowski and D. Huang, JHEP 1801 (2018) 020.

27. M.J. Baker, M. Breitbach, J. Kopp and L. Mittnacht, JHEP 1803 (2018) 114.

28. P. Zakeri, S.M.M. Nejad, M. Zakeri and S.Y. Ayazi, A minimal model for twocomponent FIMP dark matter: a basic search, arXiv:1801.09115 [hep-ph].

29. N. Bernal, C. Cosme and T. Tenkanen, Phenomenology of self-interacting dark matter in a matter-dominated universe, arXiv:1803.08064 [hep-ph].

30. N. Okada and O. Seto, Phys. Rev. D 86 (2012) 063525.

31. P.F. Perez and M.B. Wise, JHEP 1305 (2013) 094.

32. C. Cheung and Y. Zhang, JHEP 1309 (2013) 002.

33. Y. Zhang, X. Ji and R.N. Mohapatra, JHEP 1310 (2013) 104.

34. G. Bélanger, A. Goudelis, J.-C. Park and A. Pukhov, JCAP 1402 (2014) 020.

35. J.M. Cline, Z. Liu, G.D. Moore and W. Xue, Phys. Rev. D 90 (2014) 015023.

36. H. Baer, K.-Y. Choi, J.E. Kim and L. Roszkowski, Phys. Rep. 555 (2015) 1.

37. Y. Cui and B. Shuve, JHEP 1502 (2015) 049.

38. T. Appelquist et al. (LSD Collaboration), Phys. Rev. D 92 (2015) 075030.

39. J. Bramante and F. Elahi, Phys. Rev. D 91 (2015) 115001.

40. I. Baldes, N.F. Bell, A.J. Millar and R.R. Volkas, JCAP 1510 (2015) 048.

41. G. Krnjaic, Phys. Rev. D 94 (2016) 073009.

42. X.-J. Bi, Z. Kang, P. Ko, J. Li and T. Li, Phys. Rev. D 95 (2017) 043540.

43. B.W. Lee and S. Weinberg, Phys. Rev. Lett. 39 (1977) 165.

44. E.W. Kolb and M.S. Turner, The Early Universe (Westview Press, Boulder, 1994).

45. S. Dodelson, Modern Cosmology (Academic Press, San Diego, 2003).

46. P. Gondolo and G. Gelmini, Nucl. Phys. B 360 (1991) 145.

47. J. Hisano, S. Matsumoto, M. Nagai, O. Saito and M. Senami, Phys. Lett. B 646 (2007) 34.

48. M. Cirelli, A. Strumia and M. Tamburini, Nucl. Phys. B 787 (2007) 152.

49. J. March-Russell, S.M. West, D. Cumberbatch and D. Hooper, JHEP 0807 (2008) 058.

50. A. Karam and K. Tamvakis, Phys. Rev. D 94 (2016) 055004.

51. S.-Y. Guo, Z.-L. Han and Y. Liao, Phys. Rev. D 94 (2016) 115014.

52. J.A. Casas, D.G. Cerdeño, J.M. Moreno and J. Quilis, JHEP 1705 (2017) 036.

53. K. Griest and M. Kamionkowski, Phys. Rev. Lett. 64 (1990) 615.

54. S. Weinberg, The Quantum Theory of Fields Vol. I Sec. 3.7 (Cambridge University Press, Cambridge, 1995). 
55. J.D. Lewin and P.F. Smith, Astropart. Phys. 6 (1996) 87.

56. L. Vietze, P. Klos, J. Menéndez, W.C. Haxton and A. Schwenk, Phys. Rev. D 91 (2015) 043520.

57. DARWIN Collaboration (J. Aalbers et al.), JCAP 1611 (2016) 017.

58. J. Bramante, B. Broerman, R.L. Lang and N. Raj, Saturated overbuden scattering and the multiscatter frontier: discovering dark matter at the Planck mass and beyond, arXiv:1803.08044 [hep-ph].

59. M.A. Shifman, A.I. Vainshtein and V.I. Zakharov, Phys. Lett. B 78 (1978) 443.

60. J. Ellis, K.A. Olive and C. Savage, Phys. Rev. D 77 (2008) 065026.

61. A. Goudelis, B. Herrmann and O. Stål, JHEP 1309 (2013) 106.

62. F.S. Sage and R. Dick, Astropart. Phys. 71 (2015) 31.

63. M. Hoferichter, P. Klos, J. Menéndez and A. Schwenk, Phys. Rev. Lett. 119 (2017) 181803.

64. T.P. Cheng, Phys. Rev. D 38 (1988) 2869.

65. P.M. Junnarkar and A. Walker-Loud, Phys. Rev. D 87 (2013) 114510.

66. M. Gong et al. (QCD Collaboration), Phys. Rev. D 88 (2013) 014503.

67. X.-L. Ren, L.-S. Geng and J. Meng, Phys. Rev. D 91 (2015) 051502(R).

68. Y.-B. Yang, A. Alexandru, T. Draper, J. Liang and K.-F. Liu ( $\chi$ QCD Collaboration), Phys. Rev. D 94 (2016) 054503.

69. A. Abdel-Rehim, C. Alexandrou, M. Constantinou, K. Hadjiyiannakou, K. Jansen, Ch. Kallidonis, G. Koutsou and A. Vaquero Avilés-Casco (ETM Collaboration), Phys. Rev. Lett. 116 (2016) 252001.

70. G.S. Bali, S. Collins, D. Richtmann, A. Schäfer, W. Söldner, and A. Sternbeck (RQCD Collaboration), Phys. Rev. D 93 (2016) 094504.

71. J.M. Alarcón, L.S. Geng, J. Martin Camalich and J.A. Oller, Phys. Lett. B 730 (2014) 342.

72. N.F. Nasrallah and K. Schilcher, Phys. Rev. C 89 (2014) 045202.

73. S. Durr et al. (Budapest-Marseille-Wuppertal Collaboration), Phys. Rev. Lett. 116 (2016) 172001.

74. ATLAS Collaboration (G. Aad et al.), JHEP 1511 (2015) 206.

75. CMS Collaboration (V. Khatchatryan et al.), JHEP 1702 (2017) 135.

76. J.M. Alarcón, J. Martin Camalich and J.A. Oller, Phys. Rev. D 85 (2012) 051503.

77. V. Silveira and A. Zee, Phys. Lett. B 161 (1985) 136.

78. M.C. Bento, O. Bertolami, R. Rosenfeld and L. Teodoro, Phys. Rev. D 62 (2000) 041302(R).

79. C. Burgess, M. Pospelov and T. ter Veldhuis, Nucl. Phys. B 619 (2001) 709.

80. H. Davoudiasl, R. Kitano, T. Li and H. Murayama, Phys. Lett. B 609 (2005) 117.

81. R. Schabinger and J.D. Wells, Phys. Rev. D 72 (2005) 093007.

82. W.-F. Chang, J.N. Ng and J.M.S. Wu, Phys. Rev. D 74 (2006) 095005.

83. A. Kusenko, Phys. Rev. Lett. 97 (2006) 241301.

84. J. McDonald, Phys. Rev. D 50 (1994) 3637.

85. R. Dick, R.B. Mann and K.E. Wunderle, Nucl. Phys. B 805 (2008) 207.

86. C.E. Yaguna, JCAP 0903 (2009) 3.

87. A. Goudelis, Y. Mambrini and C.E. Yaguna, JCAP 0912 (2009) 8.

88. L. Lopez-Honorez and C.E. Yaguna, JCAP 1101 (2011) 002.

89. M.S. Boucenna and S. Profumo, Phys. Rev. D 84 (2011) 055011.

90. M.A. Fedderke, Jing-Yuan Chen, E.W. Kolb and Lian-Tao Wang, JCAP 1401 (2014) 001.

91. F.S. Sage and R. Dick, J. Phys. G 41 (2014) 105007.

92. L. Feng, S. Profumo and L. Ubaldi, JHEP 1503 (2015) 045. 
93. A. Beniwal, F. Rajec, C. Savage, P. Scott, C. Weniger, M. White and A.G. Williams, Phys. Rev. D 93 (2016) 115016.

94. M. Escudero, A. Berlin, D. Hooper and M.-X. Lin, JCAP 1612 (2016) 029.

95. V. Barger, P. Langacker, M. McCaskey, M.J. Ramsey-Musolf and G. Shaughnessy, Phys. Rev. D 77 (2008) 035005.

96. A. Djouadi, O. Lebedev, Y. Mambrini and J. Quevillon, Phys. Lett. B 709 (2012) 65.

97. M. Frigerio, A. Pomarol, F. Riva and A. Urbano, JHEP 1207 (2012) 015.

98. F. Bazzocchi and M. Fabbrichesi, Eur. Phys. J. C 73 (2013) 2303; Phys. Rev. D 87 (2013) 036001.

99. J.M. Cline, K. Kainulainen, P. Scott and C. Weniger, Phys. Rev. D 88 (2013) 055025; Erratum Phys. Rev. D 92 (2015) 039906.

100. A. De Simone, G.F. Giudice and A. Strumia, JHEP 1406 (2014) 081.

101. H. Han and S. Zheng, JHEP 1512 (2015) 044.

102. M. Duerr, P. Fileviez Pérez and J. Smirnov, JHEP 1606 (2016) 152.

103. S. Ghosh, A. Kundu and S. Ray, Phys. Rev. D 93 (2016) 115034.

104. H. Han, J.M. Yang, Y. Zhang and S. Zheng, Phys. Lett. B 756 (2016) 109.

105. X.-G. He and J. Tandean, JHEP 1612 (2016) 074.

106. H. Wu and S. Zheng, JHEP 1703 (2017) 142.

107. GAMBIT Collaboration (Peter Athron et al.), Eur. Phys. J. C 77 (2017) 568.

108. G. Arcadi, M. Dutra, P. Ghosh, M. Lindner, Y. Mambrini, M. Pierre, S. Profumo and F.S. Queiroz, Eur. Phys. J. C 78 (2018) 203.

109. T.G. Steele, Zhi-Wei Wang, D. Contreras and R.B. Mann, Phys. Rev. Lett. 112 (2014) 171602.

110. D.A. Demir, M. Frank and B. Korutlu, Phys. Lett. B 728 (2014) 393.

111. ATLAS Collaboration (M. Aaboud et al.), Phys. Lett. B 776 (2018) 318.

112. P.J. Fox, R. Harnik, J. Kopp and Y. Tsai, Phys. Rev. D 85 (2012) 056011.

113. A. Djouadi, A. Falkowski, Y. Mambrini and J. Quevillon, Eur. Phys. J. C 73 (2013) 2455.

114. ATLAS Collaboration (G. Aad et al.), JHEP 1511 (2015) 206.

115. F. Kahlhoefer, Int. J. Mod. Phys. A 32 (2017) 1730006.

116. O. Lebedev, H.M. Lee and Y. Mambrini, Phys. Lett. B 707 (2012) 570.

117. S. Baek, P. Ko, W.-I. Park and E. Senaha, JHEP 1305 (2013) 036.

118. C. Gross, O. Lebedev and Y. Mambrini, JHEP 1508 (2015) 158.

119. M. Duch, B. Grzadkowski and M. McGarrie, JHEP 1509 (2015) 162.

120. A. DiFranzo, P.J. Fox and T.M.P. Tait, JHEP 1604 (2016) 135.

121. C.F. Chang, X.G. He and J. Tandean, JHEP 1704 (2017) 107.

122. C. Bird, R.V. Kowalewski and M. Pospelov, Mod. Phys. Lett. A 21 (2006) 457.

123. Y.G. Kim and K.Y. Lee, Phys. Rev. D 75 (2007) 115012.

124. M. Pospelov, A. Ritz and M.B. Voloshin, Phys. Lett. B 662 (2008) 53.

125. M. Pospelov and A. Ritz, Phys. Rev. D 84 (2011) 113001.

126. L. Lopez-Honorez, T. Schwetz and J. Zupan, Phys. Lett. B 716 (2012) 179.

127. M.A. Fedderke, J.-Y. Chen, E.W. Kolb and L.-T. Wang, JHEP 1408 (2014) 122.

128. C.F. Chang, X.G. He and J. Tandean, Phys. Rev. D 96 (2017) 075026.

129. J. Billard, L. Strigari and E. Figueroa-Feliciano, Phys. Rev. D 89 (2014) 023524.

130. DEAP Collaboration (P.-A. Amaudruz et al.), Design and Construction of the DEAP-3600 Dark Matter Detector, arXiv:1712.01982 [astro-ph.IM].

131. DarkSide Collaboration (C.E. Aalseth et al.), DarkSide-20k: A 20 Tonne Two-Phase LAr TPC for Direct Dark Matter Detection at LNGS, arXiv:1707.08145 [physics.insdet]. 
132. XENON Collaboration (E. Aprile et al.), Phys. Rev. Lett. 119 (2017) 181301.

133. PandaX-II Collaboration (A. Tan et al.), Phys. Rev. Lett. 117 (2016) 121303.

134. LUX Collaboration (D. Akerib et al.), Phys. Rev. Lett. 118 (2017) 021303.

135. XENON Collaboration (E. Aprile et al.), JCAP 1604 (2016) 027.

136. LUX-ZEPLIN (LZ) Collaboration (B.J. Mount et al.), LUX-ZEPLIN (LZ) Technical Design Report, arXiv:1703.09144 [physics.ins-det]. 